\title{
Momentum Maps and Measure-valued Solutions (Peakons, Filaments and Sheets) for the EPDiff Equation
}

\author{
Darryl D. Holm* Jerrold E. Marsden ${ }^{\dagger}$ \\ To Alan Weinstein on the Occasion of his 60th Birthday \\ In The Breadth of Symplectic and Poisson Geometry \\ Progr. in Math., 232, 203-235, J. Marsden and T.S. Ratiu, Editors, \\ Birkhuser Boston, Boston, MA, 2005, A Festshrift for Alan Weinstein.
}

\begin{abstract}
This paper concerns the dynamics of measure-valued solutions of the EPDiff equations, standing for the Euler-Poincaré equations associated with the diffeomorphism group (of $\mathbb{R}^{n}$ or of an $n$-dimensional manifold $M$ ). The paper focuses on Lagrangians that are quadratic in the velocity fields and their first derivatives; that is, on geodesic motion on the diffeomorphism group with respect to a right invariant Sobolev $H^{1}$ metric. The corresponding Euler-Poincaré (EP) equations are the EPDiff equations, which coincide with the averaged template matching equations (ATME) from computer vision and agree with the Camassa-Holm $(\mathrm{CH})$ equations for shallow water waves in one dimension. The corresponding equations for the volume preserving diffeomorphism group are the LAE (Lagrangian averaged Euler) equations for incompressible fluids.

The paper shows that the EPDiff equations are generated by a smooth vector field on the diffeomorphism group for sufficiently smooth solutions. This is analogous to known results for incompressible fluids - both the Euler equations and the LAE equations - and it shows that for sufficiently smooth solutions, the equations are well-posed for short time. Numerical evidence suggests that, as time progresses, these smooth solutions break up into singular solutions which, at least in one dimension, exhibit soliton behavior.

These non-smooth, or measure-valued, solutions are higher dimensional generalizations of the peakon solutions of the $\mathrm{CH}$ equation in one dimension. One of the main purposes of the paper is to show that many of the properties of these measure-valued solutions can be understood through the fact that their solution Ansatz is a momentum map. Some additional geometry is also pointed out, for example, that this momentum map is one part of a dual pair.
\end{abstract}

${ }^{*}$ Computer and Computational Science Division, Los Alamos National Laboratory, MS D413, Los Alamos, NM 87545. email: dholm@lanl.gov and Mathematics Department, Imperial College London, SW7 2AZ, UK. email: d.holm@imperial.ac.uk

${ }^{\dagger}$ Control and Dynamical Systems 107-81, California Institute of Technology, Pasadena, CA 91125. email: marsden@cds.caltech.edu 


\section{Contents}

1 Introduction $\quad 2$

2 The EPDiff Equation $\quad 6$

3 Smoothness of the Lagrangian Equations 13

4 The Singular Solution Momentum Map $\quad 18$

5 The Geometry of the Momentum Map 23

6 Challenges, Future Directions and Speculations 28

\section{Introduction}

This paper is concerned with solutions of the EPDiff equations; that is, with the Euler-Poincaré equations associated with the diffeomorphism group in $n$-dimensions. In particular, we are concerned with singular solutions that generalize the peakon solutions of the Camassa-Holm (CH) equation from one dimension to more spatial dimensions. The CH equation (see Camassa and Holm [1993]) for the dynamics of shallow water in a certain asymptotic regime ${ }^{1}$, is

$$
u_{t}+3 u u_{x}=\alpha^{2}\left(u_{x x t}+2 u_{x} u_{x x}+u u_{x x x}\right),
$$

where $u(x, t)$ is the fluid velocity, subscripts denote partial derivatives in position $x$ and time $t$, and $\alpha^{2}$ is a positive constant, and the linear dispersion terms normally in $\mathrm{CH}$ have been omitted. Equivalently, in Hamiltonian form, this dispersionless $\mathrm{CH}$ equation reads

$$
m_{t}=-u m_{x}-2 u_{x} m=\{m, h(m)\},
$$

where $m=u-\alpha^{2} u_{x x}$ and $\alpha^{2}$ is a positive constant. As Camassa and Holm [1993] show, the $\mathrm{CH}$ equation is expressed in Hamiltonian form by using the Lie-Poisson bracket $\{\cdot, \cdot\}$ defined on the dual Lie algebra of the one dimensional vector fields and using the Hamiltonian

$$
h(m)=\frac{1}{2} \int u m d x
$$

The $\mathrm{CH}$ equation may be equivalently expressed in Euler-Poincaré form by using the Lagrangian associated with the $H^{1}$ metric for the fluid velocity. This Lagrangian is given as a function of the fluid velocity by the quadratic form

$$
l(u)=\frac{1}{2} \int\left(u^{2}+\alpha^{2} u_{x}^{2}\right) d x .
$$

\footnotetext{
${ }^{1}$ See Dullin, Gottwald and Holm [2001, 2003, 2004] for recent discussions of the derivation and asymptotic validity of the $\mathrm{CH}$ equation for shallow water waves, at one order beyond the Kortewegde Vries equation.
} 
It follows from Euler-Poincaré theory (see Marsden and Ratiu [1999] and Holm, Marsden and Ratiu [1998a]) that the one parameter curve of diffeomorphisms $\eta\left(x_{0}, t\right)$ depending on parameter $t$ and defined implicitly by

$$
\frac{\partial}{\partial t} \eta\left(x_{0}, t\right)=u\left(\eta\left(x_{0}, t\right), t\right)
$$

is a geodesic in the group of diffeomorphisms of $\mathbb{R}$ (or, with periodic boundary conditions, of the circle $S^{1}$ ) equipped with the right invariant metric equal to the $H^{1}$ metric at the identity.

A remarkable analytical property of the $\mathrm{CH}$ equation, conjectured by keeping track of derivative losses in Holm, Marsden and Ratiu [1998a] and proved in Shkoller [1998] is that the geodesic equations literally define a smooth vector field in the Sobolev $H^{s}$ topology for $s>3 / 2$. That is, in the material representation, the equations have no derivative loss. This property is analogous to the corresponding results for the Euler equations for ideal incompressible fluid flow (discovered by Ebin and Marsden [1970]) and the Lagrangian averaged Euler equations (again conjectured by Holm, Marsden and Ratiu [1998a] and proved by Shkoller [1998]).

As we will explain in $\S 3$, a similar statement holds for the $n$-dimensional EPDiff equation if we use the $H^{1}$ metric. This is all the more remarkable because smoothness of the geodesic flow is probably not true for the $L^{2}$ metric. Smoothness of volume-preserving geodesic flow with respect to the $L^{2}$ metric does hold for the incompressible flow of an ideal Euler fluid, a result proved in Ebin and Marsden [1970].

Before proceeding with a discussion of the general case of the $n$-dimensional EPDiff equations, we shall quickly review, mostly to establish notation, a few facts about the Euler-Poincaré and Lie-Poisson equations, whose basic theory is explained, for example, in Marsden and Ratiu [1999].

Review of Euler-Poincaré and Lie-Poisson Equations. Let $G$ be a Lie group and $\mathfrak{g}$ its associated Lie algebra (identified with the tangent space to $G$ at the identity element), with Lie bracket denoted $[\xi, \eta]$ for $\xi, \eta \in \mathfrak{g}$. Let $\ell: \mathfrak{g} \rightarrow \mathbb{R}$ be a given Lagrangian and let $L: T G \rightarrow \mathbb{R}$ be the right invariant Lagrangian on $G$ obtained by translating $\ell$ from the identity element to other points of $G$ via the right action of $G$ on TG. A basic result of Euler-Poincaré theory is that the Euler-Lagrange equations for $L$ on $G$ are equivalent to the (right) Euler-Poincaré equations for $\ell$ on $\mathfrak{g}$, namely to

$$
\frac{d}{d t} \frac{\delta \ell}{\delta \xi}=-\operatorname{ad}_{\xi}^{*} \frac{\delta \ell}{\delta \xi} .
$$

Here, $\operatorname{ad}_{\xi}: \mathfrak{g} \rightarrow \mathfrak{g}$ is the adjoint operator; that is, the linear map given by the Lie bracket $\eta \mapsto[\xi, \eta]$ and $\operatorname{ad}_{\xi}^{*}: \mathfrak{g}^{*} \rightarrow \mathfrak{g}^{*}$ is its dual; that is, $\left\langle\operatorname{ad}_{\xi}^{*}(\mu), \eta\right\rangle=\langle\mu,[\xi, \eta]\rangle$, where $\langle$,$\rangle denotes the natural pairing between \mathfrak{g}^{*}$ and $\mathfrak{g}$. Also, $\delta \ell / \delta \xi$ denotes the functional derivative of $\ell$ with respect to $\xi \in \mathfrak{g}$; it is defined to be the element of $\mathfrak{g}^{*}$ such that $D l(\xi) \cdot \eta=\langle\delta l / \delta \xi, \eta\rangle$ for all $\eta \in \mathfrak{g}$, where $D$ denotes the (Frechet) 
derivative $^{2}$. For left invariant systems, we change the sign of the right-hand side in (1.5). The Euler-Poincaré equations can be written in the variational form

$$
\delta \int \ell d t=0
$$

for all variations of the form $\delta \xi=\dot{\eta}-[\xi, \eta]$ for some curve $\eta$ in $\mathfrak{g}$ that vanishes at the endpoints.

If the reduced Legendre transformation $\xi \mapsto \mu=\delta \ell / \delta \xi$ is invertible, then the Euler-Poincaré equations are equivalent to the (right) Lie-Poisson equations:

$$
\dot{\mu}=-\operatorname{ad}_{\delta h / \delta \mu}^{*} \mu,
$$

where the reduced Hamiltonian is given by

$$
h(\mu)=\langle\mu, \xi\rangle-\ell(\xi) .
$$

These equations are equivalent (via Lie-Poisson reduction and reconstruction) to Hamilton's equations on $T^{*} G$ relative to the Hamiltonian $H: T^{*} G \rightarrow \mathbb{R}$, obtained by right translating $h$ from the identity element to other points via the right action of $G$ on $T^{*} G$. The Lie-Poisson equations may be written in the Poisson bracket form

$$
\dot{F}=\{F, h\},
$$

where $F: \mathfrak{g}^{*} \rightarrow \mathbb{R}$ is an arbitrary smooth function and the bracket is the (right) Lie-Poisson bracket given by

$$
\{F, G\}(\mu)=\left\langle\mu,\left[\frac{\delta F}{\delta \mu}, \frac{\delta G}{\delta \mu}\right]\right\rangle .
$$

In the important case when $\ell$ is quadratic, the Lagrangian $L$ is the quadratic form associated to a right invariant Riemannian metric on $G$. In this case, the EulerLagrange equations for $L$ on $G$ describe geodesic motion relative to this metric and these geodesics are then equivalently described by either the Euler-Poincaré, or the Lie-Poisson equations.

Outline of the paper. The main contents of the present paper are as follows:

1. In $\S 2$ we review some basic facts about the EPDiff equations, and in particular we recall a singular solution ansatz of Holm and Staley [2003, 2004] (see equation (2.8) below) that introduces a class of singular solutions that generalize the peakon solutions of the $\mathrm{CH}$ equation to higher spatial dimensions.

2. In $\S 3$ we show that the EPDiff equations possess an interesting smoothness property; namely, they define a smooth vector field (that is, they define ODE's with no derivative loss) in the Lagrangian representation. There are a number

\footnotetext{
${ }^{2}$ Of course in the finite dimensional case there is no real difference between $D l$ and $\delta l / \delta \xi$, but in the infinite dimensional case one normally does not choose $\mathfrak{g}^{*}$ to be the naive, that is, the literal functional analysis dual, but rather a convenient space for PDE needs.
} 
of interesting consequences of this; in particular, it implies that the EPDiff equations are locally well posed for sufficiently smooth initial data and that the $H^{1}$ diameter of $\operatorname{Diff}(M)$ is strictly positive. Because of the development of singularities in finite time, which the numerics suggests, the smooth solutions may not exist globally in time. This smoothness property is similar to the corresponding smoothness property of the Euler equations for ideal incompressible fluid mechanics shown in Ebin and Marsden [1970].

3. In $\S 4$, we show that the singular solution ansatz-again see (2.8) belowdefines an equivariant momentum map. We do this in a natural way by identifying the singular solutions with certain curves in the space of embeddings $\operatorname{Emb}\left(S, \mathbb{R}^{n}\right)$ of a generally lower dimensional manifold $S$ into the ambient space $\mathbb{R}^{n}$ (or an $n$-manifold $M$ ) and letting the diffeomorphism group act on this space. The right action of $\operatorname{Diff}(S)$ corresponds to the right invariance of the EPDiff equations, while the left action of $\operatorname{Diff}\left(\mathbb{R}^{n}\right)$ has a momentum map that gives the desired solution ansatz. This is the main result of the paper.

4. In $\S 5$ we briefly explore some of the geometry of the singular solution momentum map, in parallel with the corresponding work on singular solutions (vortices, filaments, etc.) for the Euler equations of an ideal fluid that was developed in Marsden and Weinstein [1983].

5. Finally, in $\S 6$, we discuss some of the remaining challenges and speculate on some of the many possible future directions for this work.

Historical Note. This paper is dedicated to our friend and collaborator Alan Weinstein and, for us, this work parallels some of our earlier collaborations with him. Alan's basic works on reduction, Poisson geometry, semidirect product theory, and stability in mechanics - just to name a few areas - have been, and remain incredibly influential and important to the field of geometric mechanics. See, for instance, Marsden and Weinstein [1974]; Weinstein [1983b]; Marsden, Ratiu and Weinstein [1984]; Weinstein [1984]; Holm, Marsden, Ratiu and Weinstein [1985].

Mechanics on Lie groups was pioneered by Arnold [1966], a reference that is a key foundation for the subject and in particular for the present paper. However, this theory was in a relatively primitive state, even by 1980, and it has benefited greatly from Alan's insights. In fact, the clear distinction between the Euler-Poincaré and Lie-Poisson equations, the former equations on $\mathfrak{g}$ and posessing a variational structure (with constraints on the variations) and the latter on $\mathfrak{g}^{*}$ with its Lie-Poisson structure took until the 1980's to crystallize, and for the place of Lie and Poincaré in the history of the subject to be clarified. This development and clarification was greatly aided by Alan's work, historical researches, and deep insight.

Alan has made key contributions to many fundamental concepts in geometric mechanics, such as Lagrangian submanifolds and related structures (Weinstein [1971, 1977]), symplectic reduction (Marsden and Weinstein [1974]), normal modes and 
periodic orbits (Weinstein [1973, 1978]), Poisson manifolds (Weinstein [1983b]), geometric phases (Weinstein [1990]), Dirac structures (Courant and Weinstein [1988]) groupoids and Lagrangian reduction (Weinstein [1996]) and the plethora of related "oid" structures he has been working on during the last decade (just look over the $151+$ papers on MathSciNet he has written!) that will surely play as an important role in the next generation of people working in the area of geometric mechanics as it is with the current new generation.

Of Alan's papers, the one that is most directly relevant to the topics discussed in the present paper is Marsden and Weinstein [1983]. Alan himself is still developing the mathematics associated with this area, as in Weinstein [2002].

\section{The EPDiff Equation}

This section reviews the EPDiff equation; that is, the Euler-Poincaré (EP) equation associated with the diffeomorphism group of an $n$-manifold $M$ (which, for simplicity, will be taken primarily as $\mathbb{R}^{n}$ ). This equation coincides with the dispersionless case of the $\mathrm{CH}$ equation for shallow water waves in one and two dimensions, discussed in Camassa and Holm [1993]; Kruse, Scheurle and Du [2001]. It also coincides with the ATME equation (the averaged template matching equation) in two dimensions. The latter equation arises in computer vision; see, for instance, Mumford [1998]; Hirani, Marsden and Arvo [2001], or Miller, Trouve and Younes [2002] for a description and further references. We have chosen to call this by a generic name, the $\boldsymbol{E P D i f f}$ equation, because it has these various interpretations in different applications. Of course these different interpretations also provide opportunities: for example, this point of view may enable one to see to what extent the singular solutions found in the EPDiff equations are applicable, either for shallow water wave interactions, or for computer vision applications. A recent combination of these ideas in which image processing is informed by concepts of momentum originating in soliton theory appears in Holm, Trouvé, Ratnanather and Younes [2004].

Statement of the EPDiff Equations. Treating analytical issues formally at this point, let $\mathfrak{X}$ denote the Lie algebra of vector fields on an $n$-dimensional manifold $M$ (such as $\mathbb{R}^{n}$ ). The space $\mathfrak{X}$ is the algebra of the diffeomorphism group of $M$, but the usual Jacobi-Lie bracket is the negative of the (standard) Lie algebra bracket. (See Marsden and Ratiu [1999] for a discussion.)

Let $\ell: \mathfrak{X} \rightarrow \mathbb{R}$ be a given Lagrangian and let $\mathfrak{M}$ denote the space of one-form densities on $M$, that is, the momentum densities. The corresponding momentum density of the fluid is defined as

$$
m=\frac{\delta \ell}{\delta u} \in \mathfrak{M}
$$

which is the functional derivative of the Lagrangian $\ell$ with respect to the fluid velocity $u \in \mathfrak{X}$. If $u$ is the basic dynamical variable, the EPDiff equations are simply the Euler-Poincaré equations associated with this Lagrangian. Equivalently, if $m$ is taken to be the basic dynamical variable, the Legendre transformation allows 
one to identify the EPDiff equations as the Lie-Poisson equations associated with the resulting Hamiltonian. For the case of $\mathbb{R}^{n}$, we will use vector notation for the momentum density $\mathbf{m}(\mathbf{x}, t): \mathbb{R}^{n} \times \mathbb{R} \rightarrow \mathbb{R}^{n}$ (a bold $\mathbf{m}$ instead of a lightface $m$ ). The EPDiff equations are as follows (see Holm, Marsden and Ratiu [1998a,b, 2002] for additional background and for techniques for computing the Euler-Poincaré equations for field theories),

$$
\frac{\partial}{\partial t} \mathbf{m}+\underbrace{\mathbf{u} \cdot \nabla \mathbf{m}}_{\text {convection }}+\underbrace{\nabla \mathbf{u}^{T} \cdot \mathbf{m}}_{\text {stretching }}+\underbrace{\mathbf{m}(\operatorname{div} \mathbf{u})}_{\text {expansion }}=0 .
$$

In coordinates $x^{i}, i=1,2, \ldots, n$, using the summation convention, and writing $\mathbf{m}=m_{i} d x^{i} \otimes d^{n} x$ (regarding $\mathbf{m}$ as a one-form density) and $\mathbf{u}=u^{i} \partial / \partial x^{i}$ (regarding $\mathbf{u}$ as a vector field), the EPDiff equations read

$$
\frac{\partial}{\partial t} m_{i}+u^{j} \frac{\partial m_{i}}{\partial x^{j}}+m_{j} \frac{\partial u^{j}}{\partial x^{i}}+m_{i} \frac{\partial u^{j}}{\partial x^{j}}=0 .
$$

The EPDiff equations can also be written concisely as

$$
\frac{\partial \mathbf{m}}{\partial t}+£_{\mathbf{u}} \mathbf{m}=0
$$

where $£_{\mathbf{u}} \mathbf{m}$ denotes the Lie derivative of the momentum one form density $\mathbf{m}$ with respect to the velocity vector field $\mathbf{u}$.

As mentioned earlier, if $\ell$ is a quadratic function of $\mathbf{u}$, then the EPDiff equation (2.1) or, equivalently, (2.3), is the Eulerian description of geodesic motion on the diffeomorphism group of the underlying space (in this case $\mathbb{R}^{n}$ ). The corresponding metric is the right invariant metric on the group, whose value on the Lie algebra (the group's tangent space at the identity - the space of vector fields) is defined by $\ell$. Since the Lagrangian $\ell$ is positive and quadratic in $\mathbf{u}$, the momentum density is linear in $\mathbf{u}$ and so defines a positive symmetric operator $Q_{\mathrm{op}}$ by

$$
\mathbf{m}=\frac{\delta \ell}{\delta \mathbf{u}}=Q_{\mathrm{op}} \mathbf{u}
$$

Likewise, for quadratic Lagrangians the velocity $\mathbf{u}$ is determined from the momentum $\mathbf{m}$ by $\mathbf{u}=G * \mathbf{m}$, where $G *$ represents convolution with the Green's function $G$ for the linear operator $Q_{\mathrm{op}}$.

Variational Formulation. Following the variational formulation of EP theory, the particular EP equation (2.1) may be derived from the following constrained variational principle:

$$
\delta \int \ell(\mathbf{u}) d t=0 .
$$

The variations are constrained to have the form

$$
\delta \mathbf{u}=\dot{\mathbf{w}}+\mathbf{w} \cdot \nabla \mathbf{u}-\mathbf{u} \cdot \nabla \mathbf{w} .
$$

This assertion may of course be verified directly. These constraints are analogous to the so-called "Lin constraints" used for a similar variational principle for fluid mechanics. (See Marsden and Ratiu [1999] for a discussion and references.) 
Hamiltonian Formulation. The Legendre transformation yields the Hamiltonian

$$
H(\mathbf{m})=\langle\mathbf{m}, \mathbf{u}\rangle-\ell(\mathbf{u}),
$$

where $\langle$,$\rangle is the natural pairing between one form densities and vector fields given$ by integration ( $L^{2}$ pairing). This Hamiltonian is the corresponding quadratic form for the momentum, namely,

$$
H(\mathbf{m})=\ell\left(Q_{\mathrm{op}}^{-1}(\mathbf{m})\right) .
$$

Of course it often happens that $Q_{\mathrm{op}}$ is a differential operator and in this case the inverse is usually given in terms of the convolution with the Green's function $G$, corresponding to the appropriate solution domain and boundary conditions;

$$
\mathbf{u}=\frac{\delta H(\mathbf{m})}{\delta \mathbf{m}}=G * \mathbf{m} .
$$

According to the general theory, the EP equation (2.1) may be expressed in Hamiltonian form by using the Lie-Poisson bracket on $\mathfrak{M}$ as

$$
\frac{\partial}{\partial t} \mathbf{m}=\{\mathbf{m}, H\}_{L P}=-\operatorname{ad}_{\delta H / \delta \mathbf{m}}^{*} \mathbf{m} .
$$

One-dimensional CH Peakon Solutions. We return now to the CH equation (1.2), which, as we have noted, is the same as the EPDiff equation (2.1) for the case of one spatial dimension, when the momentum velocity relationship is defined by the Helmholtz equation, $m=u-\alpha^{2} u_{x x}$. In one dimension, the $\mathrm{CH}$ equation has solutions whose momentum is supported at points on the real line via the following sum over Dirac delta measures,

$$
m(x, t)=\sum_{i=1}^{N} p_{i}(t) \delta\left(x-q_{i}(t)\right) .
$$

The velocity corresponding to this measure-valued momentum is obtained by convolution $u=G * m$ with the Green's function,

$$
G(|x-y|)=\frac{1}{2} e^{-|x-y| / \alpha},
$$

for the one-dimensional Helmholtz operator, $Q_{\mathrm{op}}=\left(1-\alpha^{2} \partial_{x}^{2}\right)$, appearing in the $\mathrm{CH}$ momentum velocity relationship, $m=Q_{\mathrm{op}} u$. Consequently, the $\mathrm{CH}$ velocity corresponding to this momentum is given by a superposition of peaked traveling wave pulses,

$$
u(x, t)=\frac{1}{2} \sum_{i=1}^{N} p_{i}(t) e^{-\left|x-q_{i}(t)\right| / \alpha} .
$$

Thus, the superposition of "peakons" in velocity arises from the delta function solution ansatz (2.6) for the momentum. 
Remarkably, the isospectral eigenvalue problem for the $\mathrm{CH}$ equation implies that only these singular solutions emerge asymptotically in the solution of the initial value problem in one dimension, as is shown in Camassa and Holm [1993]. Figure 2.1 shows the emergence of peakons from an initially Gaussian velocity distribution and their subsequent elastic collisions in a periodic one-dimensional domain. ${ }^{3}$ This figure demonstrates that singular solutions dominate the initial value problem. Thus, it is imperative to go beyond smooth solutions for the $\mathrm{CH}$ equation; as we shall see, there is numerical evidence that the situation is similar for the EPDiff equation.

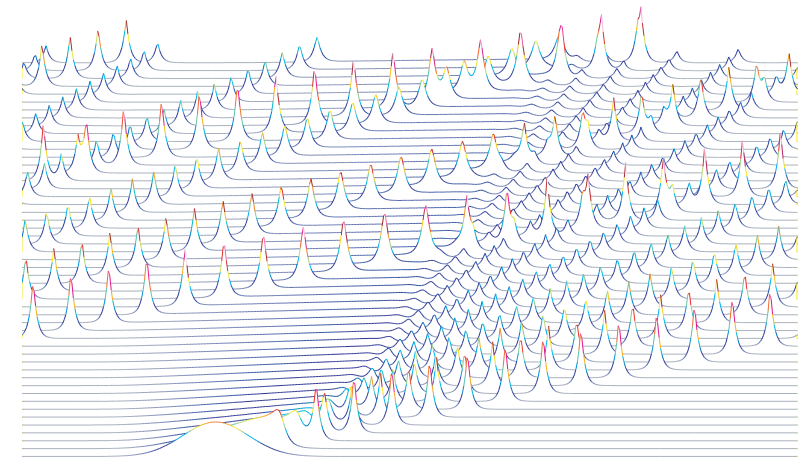

Figure 2.1: This figure shows a smooth localized (Gaussian) initial condition for the $\mathrm{CH}$ equation breaking up into an ordered train of peakons as time evolves (the time direction being vertical, which then eventually wrap around the periodic domain and interacting with other slower emergent peakons and causing a phase shift (c.f. Alber and Marsden [1992]).

Remarkably, the dynamical equations for $p_{i}(t)$ and $q_{i}(t), i=1, \ldots, N$, that arise from solution ansatz (2.6-2.7) comprise an integrable system for any $N$. This system is studied in (Alber, Camassa, Fedorov, Holm and Marsden [2001]) and references therein. See also Vaninsky [2002, 2003] for discussions of how the integrable dynamical system for $N$ peakons is related to the Toda chain with open ends.

Generalizing the $\mathrm{CH}$ peakon solutions to $n$ dimensions Building on the peakon solutions for the $\mathrm{CH}$ equation and the pulsons for its generalization to other traveling-wave shapes (see Fringer and Holm [2001]), the papers Holm and Staley [2003, 2004] introduced the following measure-valued (that is, density valued) ansatz for the $n$-dimensional solutions of the EPDiff equation (2.1):

$$
\mathbf{m}(\mathbf{x}, t)=\sum_{a=1}^{N} \int \mathbf{P}^{a}(s, t) \delta\left(\mathbf{x}-\mathbf{Q}^{a}(s, t)\right) d s .
$$

These solutions are vector-valued functions supported in $\mathbb{R}^{n}$ on a set of $N$ surfaces (or curves) of codimension $(n-k)$ for $s \in \mathbb{R}^{k}$ with $k<n$. They may, for example, be supported on sets of points (vector peakons, $k=0$ ), one-dimensional filaments (strings, $k=1$ ), or two-dimensional surfaces (sheets, $k=2$ ) in three dimensions.

\footnotetext{
${ }^{3}$ Figure 2.1 was kindly supplied by Martin Staley.
} 
One of the main results of this paper is the theorem stating that the singular solution ansatz (2.8) is an equivariant momentum map. This result helps to organize the theory and to suggest new avenues of exploration, as we shall explain.

Substitution of the solution ansatz (2.8) into the EPDiff equations (2.1) implies the following integro-partial-differential equations (IPDEs) for the evolution of such strings and sheets,

$$
\begin{aligned}
\frac{\partial}{\partial t} \mathbf{Q}^{a}(s, t) & =\sum_{b=1}^{N} \int \mathbf{P}^{b}\left(s^{\prime}, t\right) G\left(\mathbf{Q}^{a}(s, t)-\mathbf{Q}^{b}\left(s^{\prime}, t\right)\right) d s^{\prime} \\
\frac{\partial}{\partial t} \mathbf{P}^{a}(s, t) & =-\sum_{b=1}^{N} \int\left(\mathbf{P}^{a}(s, t) \cdot \mathbf{P}^{b}\left(s^{\prime}, t\right)\right) \frac{\partial}{\partial \mathbf{Q}^{a}(s, t)} G\left(\mathbf{Q}^{a}(s, t)-\mathbf{Q}^{b}\left(s^{\prime}, t\right)\right) d s^{\prime} .
\end{aligned}
$$

Importantly for the interpretation of these solutions, the coordinates $s \in \mathbb{R}^{k}$ turn out to be Lagrangian coordinates. The velocity field corresponding to the momentum solution ansatz (2.8) is given by

$$
\mathbf{u}(\mathbf{x}, t)=G * \mathbf{m}=\sum_{b=1}^{N} \int \mathbf{P}^{b}\left(s^{\prime}, t\right) G\left(\mathbf{x}-\mathbf{Q}^{b}\left(s^{\prime}, t\right)\right) d s^{\prime}, \quad \mathbf{u} \in \mathbb{R}^{n} .
$$

When evaluated along the curve $\mathbf{x}=\mathbf{Q}^{a}(s, t)$, the velocity satisfies,

$$
\left.\mathbf{u}(\mathbf{x}, t)\right|_{\mathbf{x}=\mathbf{Q}^{a}(s, t)}=\sum_{b=1}^{N} \int \mathbf{P}^{b}\left(s^{\prime}, t\right) G\left(\mathbf{Q}^{a}(s, t)-\mathbf{Q}^{b}\left(s^{\prime}, t\right)\right) d s^{\prime}=\frac{\partial \mathbf{Q}^{a}(s, t)}{\partial t} .
$$

Thus, the lower-dimensional support sets defined on $\mathbf{x}=\mathbf{Q}^{a}(s, t)$ and parameterized by coordinates $s \in \mathbb{R}^{k}$ move with the fluid velocity. Moreover, equations (2.9) for the evolution of these support sets are canonical Hamiltonian equations,

$$
\frac{\partial}{\partial t} \mathbf{Q}^{a}(s, t)=\frac{\delta H_{N}}{\delta \mathbf{P}^{a}}, \quad \frac{\partial}{\partial t} \mathbf{P}^{a}(s, t)=-\frac{\delta H_{N}}{\delta \mathbf{Q}^{a}} .
$$

The Hamiltonian function $H_{N}:\left(\mathbb{R}^{n} \times \mathbb{R}^{n}\right)^{N} \rightarrow \mathbb{R}$ is,

$$
H_{N}=\frac{1}{2} \iint \sum_{a, b=1}^{N}\left(\mathbf{P}^{a}(s, t) \cdot \mathbf{P}^{b}\left(s^{\prime}, t\right)\right) G\left(\mathbf{Q}^{a}(s, t)-\mathbf{Q}^{b}\left(s^{\prime}, t\right)\right) d s d s^{\prime} .
$$

This is the Hamiltonian for geodesic motion on the cotangent bundle of a set of curves $\mathbf{Q}^{a}(s, t)$ with respect to the metric given by $G$. This dynamics was investigated numerically in Holm and Staley [2003, 2004] to which we refer for more details of the solution properties.

As we have mentioned, one of our main goals is to show that the solution ansatz (2.8) can be recast in terms of an equivariant momentum map that naturally arises in this problem. This geometric feature underlies the remarkable reduction properties of the EPDiff equation and "explains" why the preceding equations must be 
Hamiltonian-it is because it is a general fact that equivariant momentum maps are Poisson maps.

As explained in general terms in Marsden and Weinstein [1983], the way one implements a coadjoint orbit reduction is through a momentum map, and this holds even for the case of singular orbits (again ignoring functional analytic details). Thus, in summary, the Ansatz (2.12) is the EPDiff analog of the corresponding Anzatz for incompressible fluid mechanics (that is, the EPDiff ${ }_{\mathrm{Vol}}$ equations) that gives point (or blob) vortex dynamics, vortex filaments, or sheets.

There are, however, some important differences between vortex dynamics for incompressible flows and the dynamics of the measure valued EPDiff momentum solutions. For example, the Lagrangian representations of the equations of motion show that EPDiff solutions for momentum have inertia, while the corresponding solutions for point (or blob) vortices of the EPDiff $V_{\text {Vol }}$ dynamics have no inertia. What this means is the following: the equations of motion for the spatial vectors specifying the measure valued vorticity solutions on EPDiff $\mathrm{Vol}_{\text {are }}$ first order in time, while the dynamical equations for the corresponding spatial vectors $\mathbf{Q}_{a}(s, t)$ for measure valued momentum solutions on EPDiff are second order in time. This difference has profound effects on the properties of the solutions, especially on their stability properties. Numerical investigations of Holm and Staley [2003, 2004] show, for example, that the codimension-one singular momentum solutions of EPDiff are stable, while its higher-codimension singular momentum solutions are very unstable to codimension-one perturbations. In contrast, the codimension-two singular vorticity solutions of EPDiff ${ }_{\text {Vol }}$ (point vortices in the plane, and vortex filaments in space) are known to be stable to such perturbations.

Comments on the Physical Meaning of the Equations. The EPDiff equations with the Helmholtz relation between velocity and momentum are not quite the $\mathrm{CH}$ equations for surface waves in $2 \mathrm{D}$. Those would take precisely the same form, but the shallow water wave relation in the $2 \mathrm{D} \mathrm{CH}$ approximation would be

$$
m=u-\alpha^{2} \operatorname{Grad} \operatorname{Div} u ; \quad \text { that is, } m_{i}=u_{i}-\alpha^{2} u_{j, j i}
$$

rather than the Helmholtz operator form,

$$
m=u-\alpha^{2} \operatorname{Div} \operatorname{Grad} u \quad \text { that is, } \quad m_{i}=u_{i}-\alpha^{2} u_{i, j j}
$$

The corresponding Lagrangians are, respectively,

$$
l_{\mathrm{CH}}(u)=\frac{1}{2} \int\left(|u|^{2}+\alpha^{2}(\operatorname{Div} u)^{2}\right) d x d y .
$$

and

$$
l_{\text {EPDiff }}(u)=\frac{1}{2} \int\left(|u|^{2}+\alpha^{2}|\operatorname{Grad} u|^{2}\right) d x d y .
$$

This difference was noted in Kruse, Scheurle and Du [2001], which identified (2.14) as the generalization of (1.4) for water waves in two dimensions. One may also verify this by considering the limit of the Green-Nagdhi equations for small 
potential energy. (The $\mathrm{CH}$ equation arises in this limit. The Lake and Great Lake equations of Camassa, Holm and Levermore [1996, 1997] also arise in a variant of this limit.)

Remarkably, the numerics in Holm and Staley [2003, 2004] show that the solutions for a variety of initial conditions are indistinguishable in these two cases. The initial conditions in Holm and Staley [2003, 2004] were all spatially confined velocity distributions.

Notice that this difference affects the choice of Hamiltonian, but the equations are still Euler-Poincaré equations for the diffeomorphism group and the description of the Ansatz (2.8) as a momentum map is independent of this difference in the equations.

Figure 2.2 shows the striking reconnection phenomenon seen in the nonlinear interaction between wave-trains, as simulated by numerical solutions of the EPDiff equation and observed for internal waves in the Ocean. Fig 2.2(a) shows a frame taken from simulations of the initial value problem for the EPDiff equation in two dimensions, excerpted from Holm and Staley [2003, 2004]. (See also Holm, Putkaradze and Stechmann [2003].)

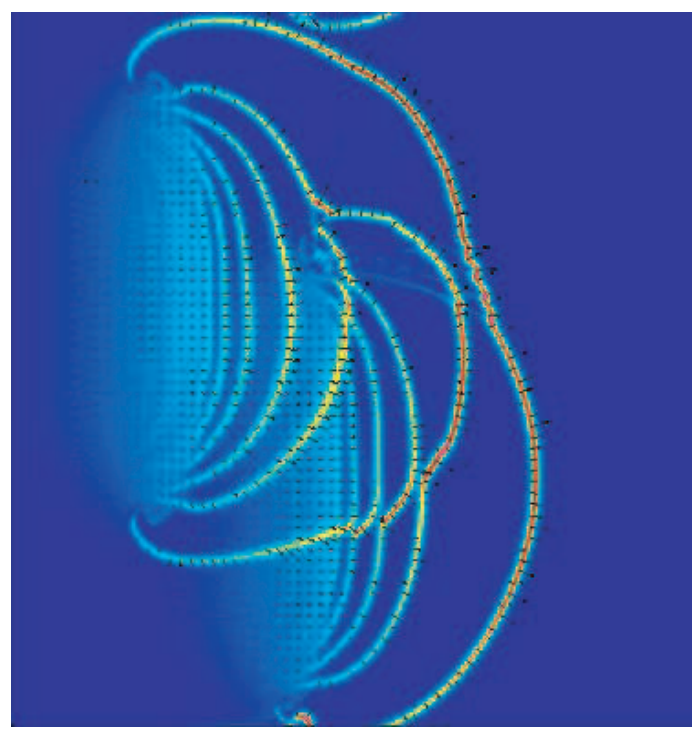

(a)

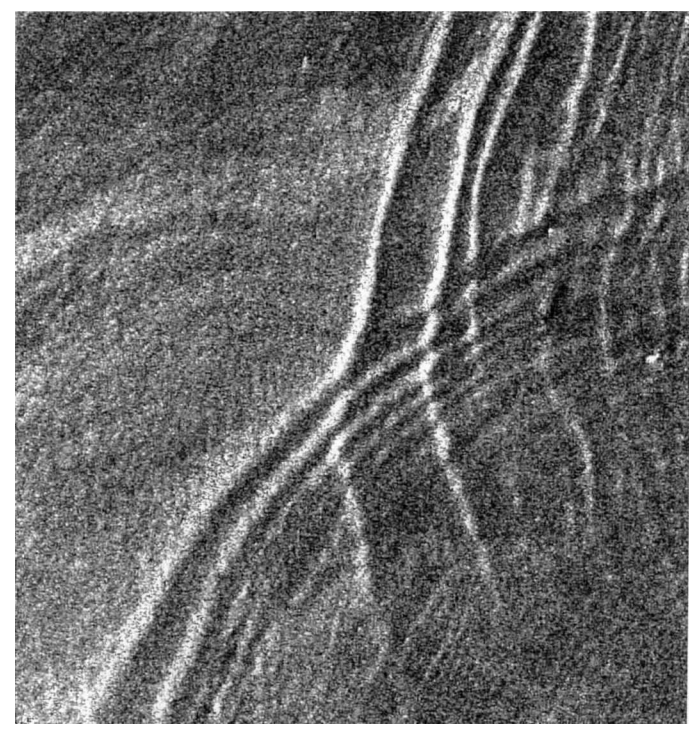

(b)

Figure 2.2: Comparison of evolutionary EPDiff solutions in two dimensions (a) and Synthetic Aperture Radar observations by the Space Shuttle of internal waves in the South China Sea (b). Both Figures show nonlinear reconnection occurring in the wave train interaction as their characteristic feature.

Fig 2.2(b) shows the interaction of two internal wave trains propagating at the interface of different density levels in the South China Sea, as observed from the Space Shuttle using synthetic aperture radar, courtesy of A. Liu (2002). Importantly, both Fig 2.2(a) and Fig 2.2(b) show nonlinear reconnection occurring in the 
wave train interaction as their characteristic feature. Fig 2.3 shows a sequence of snapshots illustrating the reconnection phenomenon for singular solutions of EPDiff in two dimensions. ${ }^{4}$
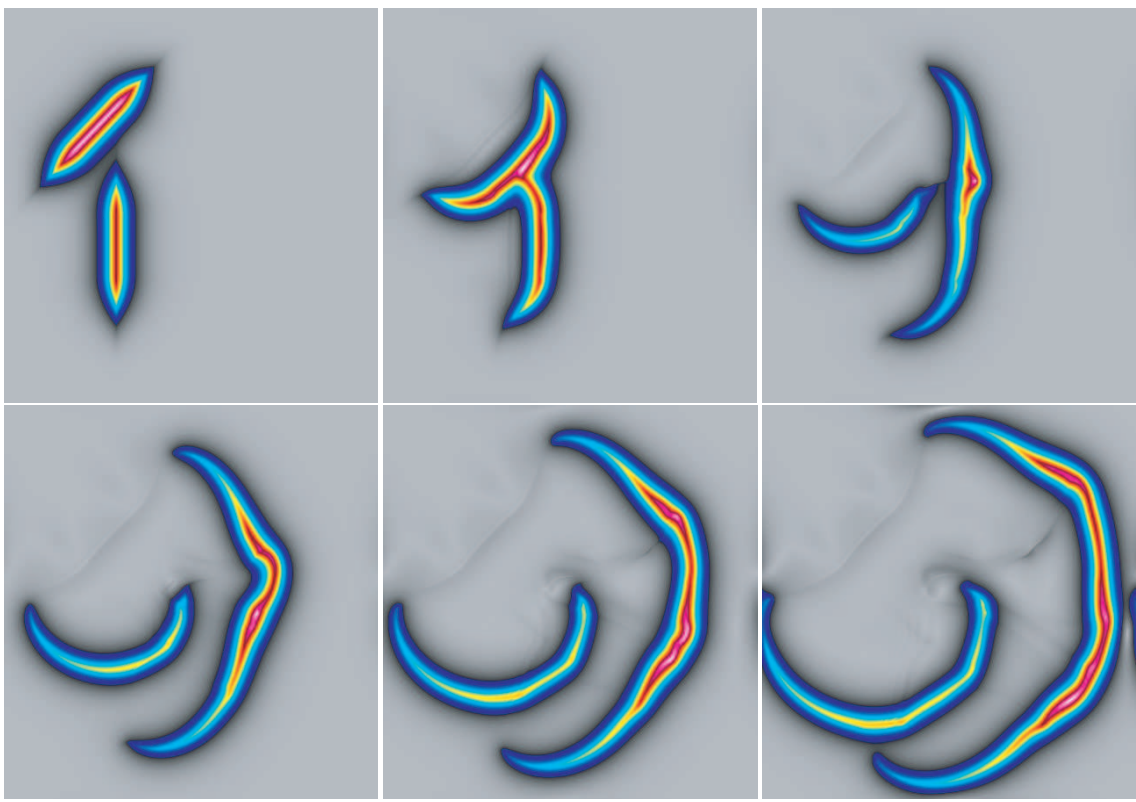

Figure 2.3: A single collision is shown to produce reconnection as the faster wave front segment initially moving Southwest along the diagonal expands, curves and obliquely overtakes the slower one, which was initially moving rightward (East). This reconnection illustrates one of the collision rules for singular solutions of the two-dimensional EPDiff flow. See Holm and Staley [2004] for a complete treatment.

Interactions among internal waves are generally thought to be described by the $\mathrm{KP}$ equation, and so any relations among the KP equation, the EPDiff equation and the 2D CH equation would be of great interest to explore; cf. Liu et al. [1998]. The derivations of the $\mathrm{KP}$ equation and the $\mathrm{CH}$ equation differ in the way the gradients of their transverse motions are treated in the asymptotics - order $O(\epsilon)$ for $\mathrm{KP}$ and order $O(1)$ for $\mathrm{CH}$; thus, some difference in their solution behavior is to be expected.

\section{Smoothness of the Lagrangian Equations}

The One-Dimensional Case. Based on a formal argument given in Holm, Marsden and Ratiu [1998a], it was shown in Shkoller [1998] that the CH equation (1.2) in Lagrangian variables defines a smooth vector field. (That is, one obtains an evolution equation with no derivative loss.) This means one can show using ODE methods that the initial value problem is well-posed and one may also establish other important properties of the equations, provided the data is sufficiently smooth.

\footnotetext{
${ }^{4}$ Fig 2.2(a) and Fig 2.3 were kindly supplied by Martin Staley.
} 
As above, we write the relation between $m$ and $u$ as $m=Q_{\mathrm{op}} u$, so that in the one dimensional case, $Q_{\mathrm{op}}$ is the operator $Q_{\mathrm{op}}=\mathrm{Id}-\alpha^{2} \partial_{x x}$. We first recall how the equations are transformed into Lagrangian variables. Introduce the one parameter curve of diffeomorphisms $\eta\left(x_{0}, t\right)$ defined implicitly by

$$
\frac{\partial}{\partial t} \eta\left(x_{0}, t\right)=u\left(\eta\left(x_{0}, t\right), t\right)
$$

so that $\eta$ is a geodesic in the group of diffeomorphisms of $\mathbb{R}$ (or, with periodic boundary conditions, of the circle $S^{1}$ ) equipped with the right invariant metric equaling the $H^{1}$ metric at the identity.

We compute the second time derivative of $\eta$ in a straightforward way by differentiating (3.1) using the chain rule:

$$
\frac{\partial^{2} \eta}{\partial t^{2}}=u u_{x}+\frac{\partial u}{\partial t}
$$

Acting on this equation with $Q_{\mathrm{op}}$ and using the definition $m=Q_{\mathrm{op}} u$ yields

$$
\begin{aligned}
Q_{\mathrm{op}} \frac{\partial^{2} \eta}{\partial t^{2}} & =Q_{\mathrm{op}}\left(u u_{x}\right)-u \partial_{x}\left(Q_{\mathrm{op}} u\right)+u m_{x}+\frac{\partial m}{\partial t} \\
& =\left[Q_{\mathrm{op}}, u \partial_{x}\right] u-2 m u_{x} \\
& =-3 \alpha^{2} u_{x} u_{x x}-2 m u_{x} \\
& =-\alpha^{2} u_{x} u_{x x}-2 u u_{x},
\end{aligned}
$$

where the third step uses the commutator relation calculated from the product rule,

$$
\left[Q_{\mathrm{op}}, u \partial_{x}\right] u=-3 \alpha^{2} u_{x} u_{x x}
$$

Hence, the preceding equation becomes

$$
\frac{\partial^{2} \eta}{\partial t^{2}}=-\frac{1}{2} Q_{\mathrm{op}}^{-1} \partial_{x}\left(\alpha^{2} u_{x}^{2}+2 u^{2}\right)
$$

The important point about this equation is that the right hand side has no derivative loss for $\alpha>0$. That is, if $u$ is in the Sobolev space $H^{s}$ for $s>5 / 2$, then the right hand is also in the same space. Regarding the right hand side as a function of $\eta$ and $\partial \eta / \partial t$, we see that it is plausible that the second order evolution equation (3.2) for $\eta$ defines a smooth ODE on the group of $H^{s}$ diffeomorphisms. (This argument requires the use of, for example, weighted Sobolev spaces in the case $x \in \mathbb{R})$.

The above is the essence of the argument given in Shkoller [1998], Remark 3.5, which in turn makes use of the type of arguments found in Ebin and Marsden [1970] for the incompressible case and which shows, by a more careful argument, that the spray is smooth if $s>3 / 2$. However, one should note that the complete argument is not quite so simple (just as in the case of incompressible fluids). A subtilty arises because smoothness means as a function of $\eta, \dot{\eta}$. Hence, one must express $u$ in terms of $\eta$, namely through the relation $u_{t}=\dot{\eta}_{t} \circ \eta_{t}^{-1}$, where the subscript $t$ here denotes 
that this argument is held fixed, and is not a partial derivative. Doing this, one sees that, while there is clearly no derivative loss, the right hand side of (3.2) does involve $\eta_{t}^{-1}$ and the map $\eta_{t} \mapsto \eta_{t}^{-1}$ is known to not be smooth. Nevertheless, the combination that appears in (3.2) is, quite remarkably, a smooth function of $\eta, \dot{\eta}$ as is shown by arguments in Ebin and Marsden [1970].

The $n$-Dimensional Case. The above argument readily generalizes to $n$-dimensions, which we shall present in the case of $\mathbb{R}^{n}$ or the flat $n$-torus $\mathbb{T}^{n}$ for simplicity. Namely, we still have the relation

$$
\frac{\partial}{\partial t} \eta\left(\mathbf{x}_{0}, t\right)=\mathbf{u}\left(\eta\left(\mathbf{x}_{0}, t\right), t\right)
$$

between $\eta$ and $\mathbf{u}$. Consequently, we may compute the second partial time derivative of $\eta$ in the usual fashion using the chain rule:

$$
\frac{\partial^{2} \eta}{\partial t^{2}}=\mathbf{u} \cdot \nabla \mathbf{u}+\frac{\partial \mathbf{u}}{\partial t}
$$

Therefore, as in the one dimensional case, we get

$$
Q_{\mathrm{op}} \frac{\partial^{2} \eta}{\partial t^{2}}=\left[Q_{\mathrm{op}},(\mathbf{u} \cdot \nabla)\right] \mathbf{u}+\mathbf{u} \cdot \nabla \mathbf{m}+\frac{\partial \mathbf{m}}{\partial t} .
$$

Calculating the commutator relation in $n$-dimensions gives

$$
\left[Q_{\mathrm{op}},(\mathbf{u} \cdot \nabla)\right] \mathbf{u}=-\alpha^{2} \operatorname{div}\left(\nabla \mathbf{u} \cdot \nabla \mathbf{u}+\nabla \mathbf{u} \cdot \nabla \mathbf{u}^{T}\right)+\alpha^{2}(\nabla \mathbf{u}) \cdot \nabla \operatorname{div} \mathbf{u}
$$

or, in components,

$$
\left(\left[Q_{\mathrm{op}},(\mathbf{u} \cdot \nabla)\right] \mathbf{u}\right)_{i}=-\alpha^{2} \partial_{k}\left(u_{i, j} u_{j, k}+u_{i, j} u_{k, j}\right)+\alpha^{2}\left(u_{i, j}\right) \partial_{j} \operatorname{div} \mathbf{u},
$$

with a sum on repeated indices.

Upon substituting the preceding commutator relation, the EPDiff equation (2.1) and the vector calculus identity

$$
-\nabla \mathbf{u}^{T} \cdot \mathbf{m}=\alpha^{2} \operatorname{div}\left(\nabla \mathbf{u}^{T} \cdot \nabla \mathbf{u}\right)-\nabla\left(\frac{1}{2}|\mathbf{u}|^{2}+\frac{\alpha^{2}}{2}|\nabla \mathbf{u}|^{2}\right),
$$

then imply the $n$-dimensional result

$$
\begin{aligned}
Q_{\mathrm{op}} \frac{\partial^{2} \eta}{\partial t^{2}}= & -\alpha^{2} \operatorname{div}\left(\nabla \mathbf{u} \cdot \nabla \mathbf{u}+\nabla \mathbf{u} \cdot \nabla \mathbf{u}^{T}-\nabla \mathbf{u}^{T} \cdot \nabla \mathbf{u}-\nabla \mathbf{u}^{T}(\operatorname{div} \mathbf{u})+\frac{1}{2} \operatorname{Id}|\nabla \mathbf{u}|^{2}\right) \\
& -\mathbf{u}(\operatorname{div} \mathbf{u})-\frac{1}{2} \nabla|\mathbf{u}|^{2} .
\end{aligned}
$$

A similar computation holds on a Riemannian manifold (or in curvilinear coordinates) in terms of covariant derivatives.

This form of the EPDiff equation is useful for interpreting some of its solution behavior. As in the one dimensional case, the crucial point is that the right hand 
side involves at most second derivatives of $\mathbf{u}$; so there is no derivative loss in the expression for $\partial^{2} \eta / \partial t^{2}$. This is the main idea behind the proof of smoothness. The technical details for the $n$-dimensional case can be provided following the arguments for the one dimensional or incompressible cases, as indicated above. This leads to the following result.

Proposition 3.1. For $s>(n / 2)+1$, and $M$ a compact Riemannian manifold, the EPDiff equations as second order equations on the $H^{s}$-Diff $(M)$ group, define a smooth spray. In particular, this implies that

1. The EPDiff equations are locally well posed (for short time) for initial data in $H^{s}$

2. Two nearby diffeomorphisms can be joined by a unique curve (lying in the neighborhood of these diffeomorphisms) which is the flow of a solution of the EPDiff equations for time ranging from 0 to 1.

3. With initial data in $H^{s}$, the solutions of the EPDiff equation are $C^{\infty}$ in time.

For example, if $M$ is the three torus, this corresponds to solutions in $\mathbb{R}^{3}$ with spatially periodic boundary conditions. By using methods such as those of Cantor [1975], we one may reasonably expect to establish a similar result for $\mathbb{R}^{n}$ in weighted (Nirenberg-Walker) Sobolev spaces.

While local well-posedness of the EPDiff equations is a fairly routine matter from the PDE point of view, the other properties are not so simple to obtain by classical PDE methods.

In the sections that follow, we will be interested in nonsmooth data. This is in stark contrast to the preceding discussion, which requires initial data that is at least $C^{1}$.

Remarkably, the same smoothness results hold for the case of the LAE- $\alpha$ (Lagrangian averaged Euler) equations, a set of incompressible equations in which small scale fluctuations whose size is of order $\alpha$ are averaged. One can view the LAE- $\alpha$ equations as the incompressible version of the EPDiff equations. This smoothness property for the LAE- $\alpha$ equations was shown by Shkoller [1998] for regions with no boundary and for regions with boundary (for various boundary conditions), it was shown in Marsden, Ratiu and Shkoller [2000]. However, unlike the incompressible case, the results apparently do not hold if $\alpha$ is zero (as was also noted in Shkoller [1998]). This sort of smoothness result also appears not to hold for many other equations, such as the KdV equation, even though it too can be realized as Euler-Poincaré equations on a Lie algebra, or as geodesics on a group, in this case the Bott-Virasoro group, as explained in Marsden and Ratiu [1999] and references therein.

The Development of Singularities. The smoothness property just discussed does not preclude the development in finite time of singular solutions from smooth localized initial data as was indicated in Figure 2.1. To capture the local singularities 
in the EPDiff solution (either verticality in slope, or discontinuities in its spatial derivative) that develop in finite time from arbitrarily smooth initial conditions, one must enlarge the solution class of interest, by considering weak solutions.

There are a number of papers on weak solutions of the $\mathrm{CH}$ equation (such as, for instance, Xin and Zhang [2000]) which we will not survey here. We just mention that the theory is not yet complete, as it is still unknown in what sense one may define global unique weak solutions to the $\mathrm{CH}$ equations in $H^{1}$-only results that have an energy conservation theorem and allow for the head-on collision of two peakons would be considered interesting. As discussed in Alber, Camassa, Fedorov, Holm and Marsden [2001] for the CH equation, one most likely must consider weak solutions in the spacetime sense.

The steepening lemma of Camassa and Holm [1993] proves that in one dimension any initial velocity distribution whose spatial profile has an inflection point with negative slope (for example, any antisymmetric smooth initial distribution of velocity on the real line) will develop a vertical slope in finite time. Note that the peakon solution (2.7) has no inflection points, so it is not subject to the steepening lemma. However, the steepening lemma underlies the mechanism for forming these singular solutions, which are continuous but have discontinuous spatial derivatives; they also lie in $H^{1}$ and have finite energy. We conclude that solutions with initial conditions in $H^{s}$ with $s>(n / 2)+1$ go to infinity in the $H^{s}$ norm in finite time, but remain in $H^{1}$ and presumably continue to exist in a weak spacetime sense for all time in $H^{1}$.

Numerical evidence in higher dimensions and the inverse scattering solution for the $\mathrm{CH}$ equation in one dimension (the latter has only discrete eigenvalues, corresponding to peakons) both suggest that the singular solutions completely dominate the time-asymptotic dynamics of the initial value problem (IVP). This singular IVP behavior is one of the main discoveries of Camassa and Holm [1993]. This singular behavior has drawn a great deal of mathematical interest to the $\mathrm{CH}$ equation and its relatives, such as EPDiff. The other properties of $\mathrm{CH}$ - its complete integrability, inverse scattering transform, connections to algebraic geometry and elliptical billiards, bi-Hamiltonian structure, etc.-are of course all interesting, too. However, the requirement of dealing with singularity as its main solution phenomenon is the primary aspect of $\mathrm{CH}$ (and EPDiff). We aim to show that many of the properties of these singular solutions of $\mathrm{CH}$ and EPDiff are captured by recognizing that the singular solution ansatz itself is a momentum map. This momentum map property explains, for example, why the singular solutions (2.8) form an invariant manifold for any value of $N$ and why their dynamics form a Hamiltonian system.

In one dimension, the complete integrability of the $\mathrm{CH}$ equation as a Hamiltonian system and its soliton paradigm explain the emergence of peakons in the $\mathrm{CH}$ dynamics. Namely, their emergence reveals the initial condition's soliton (peakon) content. However, beyond one dimension, we do not have an explicit mechanism for explaining why only singular solution behavior emerges in numerical simulations. One hopes that eventually a theory will be developed for explaining this observed singular solution phenomenon in higher dimensions. Such a theory might, for example, parallel the well-known explanation of the formation of shocks for hyperbolic 
partial differential equations. (Note, however, that EPDiff is not hyperbolic, because the relation $\mathbf{u}=G * \mathbf{m}$ between its velocity and momentum is nonlocal.)

In the remainder of this work, we shall focus our attention on the momentum map properties of the invariant manifold of singular solutions (2.8) of the EPDiff equation.

\section{The Singular Solution Momentum Map}

The Momentum Ansatz (2.8) is a Momentum Map. The purpose of this section is to show that the solution ansatz (2.8) for the momentum vector in the EPDiff equation (2.1) defines a momentum map for the action of the group of diffeomorphisms on the support sets of the Dirac delta functions. These support sets are the analogs of points on the real line for the $\mathrm{CH}$ equation in one dimension. They are points, curves, or surfaces in $\mathbb{R}^{n}$ for the EPDiff equation in $n$-dimensions.

This result, as we shall discuss in greater detail later, shows that the solution ansatz (2.8) fits naturally into the scheme of Clebsch, or canonical variables in the sense advocated by Marsden and Weinstein [1983] as well as showing that these singular solutions evolve on special coadjoint orbits for the diffeomorphism group.

One can summarize by saying that the map that implements the canonical $(\mathbf{Q}, \mathbf{P})$ variables in terms of singular solutions is a (cotangent bundle) momentum map. Such momentum maps are Poisson maps; so the canonical Hamiltonian nature of the dynamical equations for $(\mathbf{Q}, \mathbf{P})$ fits into a general theory which also provides a framework for suggesting other avenues of investigation.

Theorem 4.1. The momentum ansatz (2.8) for measure-valued solutions of the EPDiff equation (2.1), defines an equivariant momentum map

$$
\mathbf{J}_{\text {Sing }}: T^{*} \operatorname{Emb}\left(S, \mathbb{R}^{n}\right) \rightarrow \mathfrak{X}\left(\mathbb{R}^{n}\right)^{*}
$$

that we will call the singular solution momentum map.

We shall explain the notation used in this statement in the course of the proof. Right away, however, we note that the sense of "defines" is quite simple, namely expressing $\mathbf{m}$ in terms of $\mathbf{Q}, \mathbf{P}$ (which are, in turn, functions of $s$ ) can be regarded as a map from the space of $(\mathbf{Q}(s), \mathbf{P}(s))$ to the space of $\mathbf{m}$ 's.

We shall give two proofs of this result from two rather different points of view. The first proof below uses the formula for a momentum map for a cotangent lifted action, while the second proof focuses on a Poisson bracket computation. Each proof also explains the context in which one has a momentum map. (See Marsden and Ratiu [1999] for general background on momentum maps.)

First Proof. For simplicity and without loss of generality, let us take $N=1$ and so suppress the index $a$. That is, we shall take the case of an isolated singular solution. As the proof will show, this is not a real restriction.

To set the notation, fix a $k$-dimensional manifold $S$ with a given volume element and whose points are denoted $s \in S$. Let $\operatorname{Emb}\left(S, \mathbb{R}^{n}\right)$ denote the set of smooth 
embeddings $\mathbf{Q}: S \rightarrow \mathbb{R}^{n}$. (If the EPDiff equations are taken on a manifold $M$, replace $\mathbb{R}^{n}$ with $M$.) Under appropriate technical conditions, which we shall just treat formally here, $\operatorname{Emb}\left(S, \mathbb{R}^{n}\right)$ is a smooth manifold. (See, for example, Ebin and Marsden [1970] and Marsden and Hughes [1983] for a discussion and references.)

The tangent space $T_{\mathbf{Q}} \operatorname{Emb}\left(S, \mathbb{R}^{n}\right)$ to $\operatorname{Emb}\left(S, \mathbb{R}^{n}\right)$ at the point $\mathbf{Q} \in \operatorname{Emb}\left(S, \mathbb{R}^{n}\right)$ is given by the space of material velocity fields, namely the linear space of maps $\mathbf{V}: S \rightarrow \mathbb{R}^{n}$ that are vector fields over the map $\mathbf{Q}$. The dual space to this space will be identified with the space of one-form densities over $\mathbf{Q}$, which we shall regard as maps $\mathbf{P}: S \rightarrow\left(\mathbb{R}^{n}\right)^{*}$. In summary, the cotangent bundle $T^{*} \operatorname{Emb}\left(S, \mathbb{R}^{n}\right)$ is identified with the space of pairs of maps $(\mathbf{Q}, \mathbf{P})$.

These give us the domain space for the singular solution momentum map. Now we consider the action of the symmetry group. Consider the group $\mathfrak{G}=$ Diff of diffeomorphisms of the space $M$ in which the EPDiff equations are operating, concretely in our case $\mathbb{R}^{n}$. Let it act on $M$ by composition on the left. Namely for $\eta \in \operatorname{Diff}\left(\mathbb{R}^{n}\right)$, we let

$$
\eta \cdot \mathbf{Q}=\eta \circ \mathbf{Q} .
$$

Now lift this action to the cotangent bundle $T^{*} \operatorname{Emb}\left(S, \mathbb{R}^{n}\right)$ in the standard way (see, for instance, Marsden and Ratiu [1999] for this construction). This lifted action is a symplectic (and hence Poisson) action and has an equivariant momentum map. We claim that this momentum map is precisely given by the ansatz (2.8).

To see this, we will recall and then apply the general formula for the momentum map associated with an action of a general Lie group $\mathfrak{G}$ on a configuration manifold $Q$ and cotangent lifted to $T^{*} Q$.

First let us recall the general formula. Namely, the momentum map is the map $\mathbf{J}: T^{*} Q \rightarrow \mathfrak{g}^{*}\left(\mathfrak{g}^{*}\right.$ denotes the dual of the Lie algebra $\mathfrak{g}$ of $\left.\mathfrak{G}\right)$ defined by

$$
\mathbf{J}\left(\alpha_{q}\right) \cdot \xi=\left\langle\alpha_{q}, \xi_{Q}(q)\right\rangle
$$

where $\alpha_{q} \in T_{q}^{*} Q$ and $\xi \in \mathfrak{g}$, where $\xi_{Q}$ is the infinitesimal generator of the action of $\mathfrak{G}$ on $Q$ associated to the Lie algebra element $\xi$, and where $\left\langle\alpha_{q}, \xi_{Q}(q)\right\rangle$ is the natural pairing of an element of $T_{q}^{*} Q$ with an element of $T_{q} Q$.

Now we apply formula (4.2) to the special case in which the group $\mathfrak{G}$ is the diffeomorphism group $\operatorname{Diff}\left(\mathbb{R}^{n}\right)$, the manifold $Q$ is $\operatorname{Emb}\left(S, \mathbb{R}^{n}\right)$ and where the action of the group on $\operatorname{Emb}\left(S, \mathbb{R}^{n}\right)$ is given by (4.1). The sense in which the Lie algebra of $\mathfrak{G}=$ Diff is the space $\mathfrak{g}=\mathfrak{X}$ of vector fields is well-understood. Hence, its dual is naturally regarded as the space of one-form densities. The momentum map is thus a map $\mathbf{J}: T^{*} \operatorname{Emb}\left(S, \mathbb{R}^{n}\right) \rightarrow \mathfrak{X}^{*}$.

To calculate $\mathbf{J}$ given by (4.2), we first work out the infinitesimal generators. Let $X \in \mathfrak{X}$ be a Lie algebra element. By differentiating the action (4.1) with respect to $\eta$ in the direction of $X$ at the identity element we find that the infinitesimal generator is given by

$$
X_{\operatorname{Emb}\left(S, \mathbb{R}^{n}\right)}(\mathbf{Q})=X \circ \mathbf{Q}
$$


Thus, taking $\alpha_{q}$ to be the cotangent vector $(\mathbf{Q}, \mathbf{P})$, equation (4.2) gives

$$
\begin{aligned}
\langle\mathbf{J}(\mathbf{Q}, \mathbf{P}), X\rangle & =\langle(\mathbf{Q}, \mathbf{P}), X \circ \mathbf{Q}\rangle \\
& =\int_{S} P_{i}(s) X^{i}(\mathbf{Q}(s)) d^{k} s .
\end{aligned}
$$

On the other hand, note that the right hand side of (2.8) (again with the index $a$ suppressed, and with $t$ suppressed as well), when paired with the Lie algebra element $X$ is

$$
\begin{aligned}
\left\langle\int_{S} \mathbf{P}(s) \delta(\mathbf{x}-\mathbf{Q}(s)) d^{k} s, X\right\rangle & =\int_{\mathbb{R}^{n}} \int_{S}\left(P_{i}(s) \delta(\mathbf{x}-\mathbf{Q}(s)) d^{k} s\right) X^{i}(\mathbf{x}) d^{n} x \\
& =\int_{S} P_{i}(s) X^{i}\left(\mathbf{Q}(s) d^{k} s .\right.
\end{aligned}
$$

This shows that the expression given by (2.8) is equal to $\mathbf{J}$ and so the result is proved.

Second Proof. As is standard (see, for example, Marsden and Ratiu [1999]), one can characterize momentum maps by means of the following relation, required to hold for all functions $F$ on $T^{*} \operatorname{Emb}\left(S, \mathbb{R}^{n}\right)$; that is, functions of $\mathbf{Q}$ and $\mathbf{P}$ :

$$
\{F,\langle\mathbf{J}, \xi\rangle\}=\xi_{P}[F] .
$$

In our case, we shall take $\mathbf{J}$ to be given by the solution ansatz and verify that it satisfies this relation. To do so, let $\xi \in \mathfrak{X}$ so that the left side of (4.3) becomes

$$
\left\{F, \int_{S} P_{i}(s) \xi^{i}(\mathbf{Q}(s)) d^{k} s\right\}=\int_{S}\left[\frac{\delta F}{\delta Q^{i}} \xi^{i}(\mathbf{Q}(s))-P_{i}(s) \frac{\delta F}{\delta P_{j}} \frac{\delta}{\delta Q^{j}} \xi^{i}(\mathbf{Q}(s))\right] d^{k} s .
$$

On the other hand, one can directly compute from the definitions that the infinitesimal generator of the action on the space $T^{*} \operatorname{Emb}\left(S, \mathbb{R}^{n}\right)$ corresponding to the vector field $\xi^{i}(\mathbf{x}) \frac{\partial}{\partial Q^{i}}$ (a Lie algebra element), is given by (see Marsden and Ratiu [1999], formula (12.1.14)):

$$
\delta \mathbf{Q}=\xi \circ \mathbf{Q}, \quad \delta \mathbf{P}=-P_{i}(s) \frac{\partial}{\partial \mathbf{Q}} \xi^{i}(\mathbf{Q}(s)),
$$

which verifies that (4.3) holds.

An important element left out in this proof so far is that it does not make clear that the momentum map is equivariant, a condition needed for the momentum map to be Poisson. The first proof took care of this automatically since momentum maps for cotangent lifted actions are always equivariant and hence Poisson.

Thus, to complete the second proof, we need to check directly that the momentum map is equivariant. Actually, we shall only check that it is infinitesimally invariant by showing that it is a Poisson map from $T^{*} \operatorname{Emb}\left(S, \mathbb{R}^{n}\right)$ to the space of m's (the dual of the Lie algebra of $\mathfrak{X}$ ) with its Lie-Poisson bracket. This sort of 
approach to characterize equivariant momentum maps is discussed in an interesting way in Weinstein [2002].

The following computation accomplishes this methodology by showing directly that the singular solution momentum map is Poisson. To do this, we use the canonical Poisson brackets for $\{\mathbf{P}\},\{\mathbf{Q}\}$ and apply the chain rule to compute $\left\{m_{i}(\mathbf{x}), m_{j}(\mathbf{y})\right\}$. Using the notation $\delta_{k}^{\prime}(\mathbf{y}) \equiv \partial \delta(\mathbf{y}) / \partial y^{k}$, we get

$$
\begin{aligned}
& \left\{m_{i}(\mathbf{x}), m_{j}(\mathbf{y})\right\} \\
& =\left\{\sum_{a=1}^{N} \int d s P_{i}^{a}(s, t) \delta\left(\mathbf{x}-\mathbf{Q}^{a}(s, t)\right), \sum_{b=1}^{N} \int d s^{\prime} P_{j}^{b}\left(s^{\prime}, t\right) \delta\left(\mathbf{y}-\mathbf{Q}^{b}\left(s^{\prime}, t\right)\right)\right\} \\
& =\sum_{a, b=1}^{N} \iint d s d s^{\prime}\left[\left\{P_{i}^{a}(s), P_{j}^{b}\left(s^{\prime}\right)\right\} \delta\left(\mathbf{x}-\mathbf{Q}^{a}(s)\right) \delta\left(\mathbf{y}-\mathbf{Q}^{b}\left(s^{\prime}\right)\right)\right. \\
& \quad-\left\{P_{i}^{a}(s), Q_{k}^{b}\left(s^{\prime}\right)\right\} P_{j}^{b}\left(s^{\prime}\right) \delta\left(\mathbf{x}-\mathbf{Q}^{a}(s)\right) \delta_{k}^{\prime}\left(\mathbf{y}-\mathbf{Q}^{b}\left(s^{\prime}\right)\right) \\
& \quad-\left\{Q_{k}^{a}(s), P_{j}^{b}\left(s^{\prime}\right)\right\} P_{i}^{a}(s) \delta_{k}^{\prime}\left(\mathbf{x}-\mathbf{Q}^{a}(s)\right) \delta\left(\mathbf{y}-\mathbf{Q}^{b}\left(s^{\prime}\right)\right) \\
& \left.\quad+\left\{Q_{k}^{a}(s), Q_{\ell}^{b}\left(s^{\prime}\right)\right\} P_{i}^{a}(s) P_{j}^{b}\left(s^{\prime}\right) \delta_{k}^{\prime}\left(\mathbf{x}-\mathbf{Q}^{a}(s)\right) \delta_{\ell}^{\prime}\left(\mathbf{y}-\mathbf{Q}^{b}\left(s^{\prime}\right)\right)\right] .
\end{aligned}
$$

Substituting the canonical Poisson bracket relations

$$
\begin{aligned}
& \left\{P_{i}^{a}(s), P_{j}^{b}\left(s^{\prime}\right)\right\}=0 \\
& \left\{Q_{k}^{a}(s), Q_{\ell}^{b}\left(s^{\prime}\right)\right\}=0, \quad \text { and } \\
& \left\{Q_{k}^{a}(s), P_{j}^{b}\left(s^{\prime}\right)\right\}=\delta^{a b} \delta_{k j} \delta\left(s-s^{\prime}\right)
\end{aligned}
$$

into the preceding computation yields,

$$
\begin{aligned}
\left\{m_{i}(\mathbf{x}), m_{j}(\mathbf{y})\right\} & \\
= & \left\{\sum_{a=1}^{N} \int d s P_{i}^{a}(s, t) \delta\left(\mathbf{x}-\mathbf{Q}^{a}(s, t)\right), \sum_{b=1}^{N} \int d s^{\prime} P_{j}^{b}\left(s^{\prime}, t\right) \delta\left(\mathbf{y}-\mathbf{Q}^{b}\left(s^{\prime}, t\right)\right)\right\} \\
= & \sum_{a=1}^{N} \int d s P_{j}^{a}(s) \delta\left(\mathbf{x}-\mathbf{Q}^{a}(s)\right) \delta_{i}^{\prime}\left(\mathbf{y}-\mathbf{Q}^{a}(s)\right) \\
& -\sum_{a=1}^{N} \int d s P_{i}^{a}(s) \delta_{j}^{\prime}\left(\mathbf{x}-\mathbf{Q}^{a}(s)\right) \delta\left(\mathbf{y}-\mathbf{Q}^{a}(s)\right) \\
= & -\left(m_{j}(\mathbf{x}) \frac{\partial}{\partial x^{i}}+\frac{\partial}{\partial x^{j}} m_{i}(\mathbf{x})\right) \delta(\mathbf{x}-\mathbf{y}) .
\end{aligned}
$$

Thus,

$$
\left\{m_{i}(\mathbf{x}), m_{j}(\mathbf{y})\right\}=-\left(m_{j}(\mathbf{x}) \frac{\partial}{\partial x^{i}}+\frac{\partial}{\partial x^{j}} m_{i}(\mathbf{x})\right) \delta(\mathbf{x}-\mathbf{y}),
$$

which is readily checked to be the Lie-Poisson bracket on the space of $m$ 's. This completes the second proof of theorem.

Each of these proofs has shown the following basic fact. 
Corollary 4.2. The singular solution momentum map defined by the singular solution ansatz, namely,

$$
\mathbf{J}_{\text {Sing }}: T^{*} \operatorname{Emb}\left(S, \mathbb{R}^{n}\right) \rightarrow \mathfrak{X}\left(\mathbb{R}^{n}\right)^{*}
$$

is a Poisson map from the canonical Poisson structure on $T^{*} \operatorname{Emb}\left(S, \mathbb{R}^{n}\right)$ to the Lie-Poisson structure on $\mathfrak{X}\left(\mathbb{R}^{n}\right)^{*}$.

This is perhaps the most basic property of the singular solution momentum map. Some of its more sophisticated properties are outlined in the following section.

Pulling Back the Equations. Since the solution ansatz (2.8) has been shown in the preceding Corollary to be a Poisson map, the pull back of the Hamiltonian from $\mathfrak{X}^{*}$ to $T^{*} \operatorname{Emb}\left(S, \mathbb{R}^{n}\right)$ gives equations of motion on the latter space that project to the equations on $\mathfrak{X}^{*}$.

Thus, the basic fact that the momentum map $\mathbf{J}_{\text {Sing }}$ is Poisson explains why the functions $\mathbf{Q}^{a}(s, t)$ and $\mathbf{P}^{a}(s, t)$ satisfy canonical Hamiltonian equations.

Note that the coordinate $s \in \mathbb{R}^{k}$ that labels these functions is a "Lagrangian coordinate" in the sense that it does not evolve in time but rather labels the solution.

In terms of the pairing

$$
\langle\cdot, \cdot\rangle: \mathfrak{g}^{*} \times \mathfrak{g} \rightarrow \mathbb{R}
$$

between the Lie algebra $\mathfrak{g}$ (vector fields in $\mathbb{R}^{n}$ ) and its dual $\mathfrak{g}^{*}$ (one-form densities in $\mathbb{R}^{n}$ ), the following relation holds for measure-valued solutions under the momentum map (2.8),

$$
\begin{aligned}
\langle\mathbf{m}, \mathbf{u}\rangle & =\int \mathbf{m} \cdot \mathbf{u} d^{n} \mathbf{x}, \quad L^{2} \text { pairing for } \mathbf{m} \& \mathbf{u} \in \mathbb{R}^{n} \\
& =\iint \sum_{a, b=1}^{N}\left(\mathbf{P}^{a}(s, t) \cdot \mathbf{P}^{b}\left(s^{\prime}, t\right)\right) G\left(\mathbf{Q}^{a}(s, t)-\mathbf{Q}^{b}\left(s^{\prime}, t\right)\right) d s d s^{\prime} \\
& =\int \sum_{a=1}^{N} \mathbf{P}^{a}(s, t) \cdot \frac{\partial \mathbf{Q}^{a}(s, t)}{\partial t} d s \\
& \equiv\langle\langle\mathbf{P}, \dot{\mathbf{Q}}\rangle,
\end{aligned}
$$

which is the natural pairing between the points $(\mathbf{Q}, \mathbf{P}) \in T^{*} \operatorname{Emb}\left(S, \mathbb{R}^{n}\right)$ and $(\mathbf{Q}, \dot{\mathbf{Q}}) \in$ $T \operatorname{Emb}\left(S, \mathbb{R}^{n}\right)$.

The pull-back of the Hamiltonian $H[\mathbf{m}]$ defined on the dual of the Lie algebra $\mathfrak{g}^{*}$, to $T^{*} \operatorname{Emb}\left(S, \mathbb{R}^{n}\right)$ is easily seen to be consistent with what we had before:

$$
H[\mathbf{m}] \equiv \frac{1}{2}\langle\mathbf{m}, G * \mathbf{m}\rangle=\frac{1}{2}\langle\langle\mathbf{P}, G * \mathbf{P}\rangle\rangle \equiv H_{N}[\mathbf{P}, \mathbf{Q}] .
$$

In summary, in concert with the Poisson nature of the singular solution momentum map, we see that the singular solutions in terms of $\mathbf{Q}$ and $\mathbf{P}$ satisfy Hamiltonian equations and also define an invariant solution set for the EPDiff equations. In fact, 
This invariant solution set is a special coadjoint orbit for the diffeomorphism group, as we shall discuss in the next section.

Remark. It would be extremely interesting if the smoothness properties explored in $\S 3$ were also valid on the space $T^{*} \operatorname{Emb}\left(S, \mathbb{R}^{n}\right)$. This is obviously valid for the $\mathrm{CH}$ equation.

\section{The Geometry of the Momentum Map}

In this section we explore the geometry of the singular solution momentum map discussed in $\S 4$ in a little more detail. The approach may be stated as follows: simply apply all of the ideas given in Marsden and Weinstein [1983] in a systematic way to the current setting. As in that paper, the treatment is formal, in the sense that there are a number of technical issues in the infinite dimensional case that are left open. We will mention a few of these as we proceed.

Coadjoint Orbits. We claim that the image of the singular solution momentum map is a coadjoint orbit in $\mathfrak{X}^{*}$. This means that (modulo some issues of connectedness and smoothness, which we do not consider here) the solution ansatz given by (2.8) defines a coadjoint orbit in the space of all one-form densities, regarded as the dual of the Lie algebra of the diffeomorphism group. These coadjoint orbits should be thought of as singular orbits - that is, due to their special nature, they are not generic.

Recognizing them as coadjoint orbits is one way of gaining further insight into why the singular solutions form dynamically invariant sets - it is a general fact that coadjoint orbits in $\mathfrak{g}^{*}$ are symplectic submanifolds of the Lie-Poisson manifold $\mathfrak{g}^{*}$ (in our case $\mathfrak{X}\left(\mathbb{R}^{n}\right)^{*}$ ) and, correspondingly, are dynamically invariant for any Hamiltonian system on $\mathfrak{g}^{*}$.

The idea of the proof of our claim is simply this: whenever one has an equivariant momentum map $\mathbf{J}: P \rightarrow \mathfrak{g}^{*}$ for the action of a group $G$ on a symplectic or Poisson manifold $P$, and that action is transitive, then the image of $\mathbf{J}$ is an orbit (or at least a piece of an orbit). This general result, due to Kostant, is stated more precisely in Marsden and Ratiu [1999], Theorem 14.4.5. Roughly speaking, the reason that transitivity holds in our case is because one can "move the images of the manifolds $S$ around at will with arbitrary velocity fields" using diffeomorphisms of $\mathbb{R}^{n}$.

Symplectic Structure on Orbits. Recall (from, for example, Marsden and Ratiu [1999]), the general formula for the symplectic structure on coadjoint orbits:

$$
\Omega_{\mu}\left(\xi_{\mathfrak{g}^{*}}(\mu), \eta_{\mathfrak{g}^{*}}(\mu)\right)=\langle\mu,[\xi, \eta]\rangle
$$

where $\mu \in \mathfrak{g}^{*}$ is a chosen point on an orbit and where $\xi, \eta$ are elements of $\mathfrak{g}$. We use a plus sign in this formula since we are dealing with orbits for the right action. 
Just as in Marsden and Weinstein [1983], this line of investigation leads to an explicit formula for the coadjoint orbit symplectic structure in the case of Diff. In the present case, it is a particularly simple and transparent formula.

Recall that in the case of incompressible fluid mechanics, this procedure leads naturally to the symplectic (and Poisson) structure for many interesting singular coadjoint orbits, such as point vortices in the plane, vortex patches, vortex blobs (closely related to the planar LAE- $\alpha$ equations) and vortex filaments. An important point is that this structure is independent of how these solutions are parametrized.

For the case of the diffeomorphism group, let $\mathcal{O}_{\mathbf{m}}$ denote the coadjoint orbit through the point $\mathbf{m} \in \mathfrak{X}^{*}\left(\mathbb{R}^{n}\right)$.

Theorem 5.1. The symplectic structure $\Omega_{\mathbf{m}}$ on $T_{\mathbf{m}} \mathcal{O}_{\mathbf{m}}$ is given by

$$
\Omega_{\mathbf{m}}\left(£_{u_{1}} \mathbf{m}, £_{u_{2}} \mathbf{m}\right)=-\int\left\langle\mathbf{m},\left[u_{1}, u_{2}\right]\right\rangle d^{n} x
$$

Proof. We substitute into the general Kirillov-Kostant-Souriau formula (5.1) for the symplectic structure on coadjoint orbits. (As noted above, there is a + sign, since we are dealing with a right invariant system). The only thing needing explanation is that our Lie algebra convention always uses the left Lie bracket. For Diff, this is the negative of the usual Lie bracket, as is explained in Marsden and Ratiu [1999].

The Momentum map $\mathbf{J}_{S}$ and the Kelvin circulation theorem. The momentum map $\mathbf{J}_{\text {Sing }}$ involves $\operatorname{Diff}\left(\mathbb{R}^{n}\right)$, the left action of the diffeomorphism group on the space of embeddings $\operatorname{Emb}\left(S, \mathbb{R}^{n}\right)$ by smooth maps of the target space $\mathbb{R}^{n}$, namely,

$$
\operatorname{Diff}\left(\mathbb{R}^{n}\right): \mathbf{Q} \cdot \eta=\eta \circ \mathbf{Q},
$$

where, recall, Q $: S \rightarrow \mathbb{R}^{n}$. As above, the cotangent bundle $T^{*} \operatorname{Emb}\left(S, \mathbb{R}^{n}\right)$ is identified with the space of pairs of maps $(\mathbf{Q}, \mathbf{P})$, with $\mathbf{Q}: S \rightarrow \mathbb{R}^{n}$ and $\mathbf{P}: S \rightarrow$ $T^{*} \mathbb{R}^{n}$.

However, there is another momentum map $\mathbf{J}_{S}$ associated with the right action of the diffeomorphism group of $S$ on the embeddings $\operatorname{Emb}\left(S, \mathbb{R}^{n}\right)$ by smooth maps of the "Lagrangian labels" $S$ (fluid particle relabeling by $\eta: S \rightarrow S$ ). This action is given by

$$
\operatorname{Diff}(S): \mathbf{Q} \cdot \eta=\mathbf{Q} \circ \eta .
$$

The infinitesimal generator of this right action is

$$
X_{\operatorname{Emb}\left(S, \mathbb{R}^{n}\right)}(\mathbf{Q})=\left.\frac{d}{d t}\right|_{t=0} \mathbf{Q} \circ \eta_{t}=T \mathbf{Q} \circ X .
$$

where $X \in \mathfrak{X}$ is tangent to the curve $\eta_{t}$ at $t=0$. Thus, again taking $N=1$ (so we suppress the index $a$ ) and also letting $\alpha_{q}$ in the momentum map formula (4.2) be 
the cotangent vector $(\mathbf{Q}, \mathbf{P})$, one computes $\mathbf{J}_{S}$ :

$$
\begin{aligned}
\left\langle\mathbf{J}_{S}(\mathbf{Q}, \mathbf{P}), X\right\rangle & =\langle(\mathbf{Q}, \mathbf{P}), T \mathbf{Q} \cdot X\rangle \\
& =\int_{S} P_{i}(s) \frac{\partial Q^{i}(s)}{\partial s^{m}} X^{m}(s) d^{k} s \\
& =\int_{S} X(\mathbf{P}(s) \cdot d \mathbf{Q}(s)) d^{k} s \\
& =\left(\int_{S} \mathbf{P}(s) \cdot d \mathbf{Q}(s) \otimes d^{k} s, X(s)\right) \\
& =\langle\mathbf{P} \cdot d \mathbf{Q}, X\rangle .
\end{aligned}
$$

Consequently, the momentum map formula (4.2) yields

$$
\mathbf{J}_{S}(\mathbf{Q}, \mathbf{P})=\mathbf{P} \cdot d \mathbf{Q}
$$

with the indicated pairing of the one-form density $\mathbf{P} \cdot d \mathbf{Q}$ with the vector field $X$.

We have set things up so that the following is true.

Proposition 5.2. The momentum map $\mathbf{J}_{S}$ is preserved by the evolution equations (2.12) for $\mathbf{Q}$ and $\mathbf{P}$.

Proof. It is enough to notice that the Hamiltonian $H_{N}$ in equation (2.13) is invariant under the cotangent lift of the action of $\operatorname{Diff}(S)$; it merely amounts to the invariance of the integral over $S$ under reparametrization; that is, the change of variables formula; keep in mind that $\mathbf{P}$ includes a density factor.

This result is similar to the Kelvin-Noether theorem for circulation $\Gamma$ of an ideal fluid, which may be written as $\Gamma=\oint_{c(s)} D(s)^{-1} \mathbf{P}(s) \cdot d \mathbf{Q}(s)$ for each Lagrangian circuit $c(s)$, where $D$ is the mass density and $\mathbf{P}$ is again the canonical momentum density. This similarity should come as no surprise, because the Kelvin-Noether theorem for ideal fluids arises from invariance of Hamilton's principle under fluid parcel relabeling by the same right action of the diffeomorphism group, as in (5.3).

Note that, being an equivariant momentum map, the map $\mathbf{J}_{S}$, as with $\mathbf{J}_{\text {Sing, }}$, is also a Poisson map. That is, substituting the canonical Poisson bracket into relation (5.5); that is, the relation $\mathbf{M}(\mathbf{x})=\sum_{i} P_{i}(\mathbf{x}) \nabla Q^{i}(\mathbf{x})$ yields the Lie-Poisson bracket on the space of $\mathbf{M}$ 's. We use the different notations $\mathbf{m}$ and $\mathbf{M}$ because these quantitites are analogous to the body and spatial angular momentum for rigid body mechanics. In fact, the quantity $\mathbf{m}$ given by the solution Ansatz; specifically, $\mathbf{m}=\mathbf{J}_{\text {Sing }}(\mathbf{Q}, \mathbf{P})$ gives the singular solutions of the EPDiff equations, while $\mathbf{M}(\mathbf{x})=$ $\mathbf{J}_{S}(\mathbf{Q}, \mathbf{P})=\sum_{i} P_{i}(\mathbf{x}) \nabla Q^{i}(\mathbf{x})$ is a conserved quantity.

In the language of fluid mechanics, the expression of $\mathbf{m}$ in terms of $(\mathbf{Q}, \mathbf{P})$ is an example of a "Clebsch representation," which expresses the solution of the EPDiff equations in terms of canonical variables that evolve by standard canonical Hamilton equations. This has been known in the case of fluid mechanics for more than 100 
years. For modern discussions of the Clebsch representation for ideal fluids, see, for example, Holm and Kupershmidt [1983]; Marsden and Weinstein [1983].

One more remark is in order; namely the special case in which $S=M$ is of course allowed. In this case, $\mathbf{Q}$ corresponds to the map $\eta$ itself and $\mathbf{P}$ just corresponds to its conjugate momentum. The quantity $\mathbf{m}$ corresponds to the spatial (dynamic) momentum density (that is, right translation of $\mathbf{P}$ to the identity), while $\mathbf{M}$ corresponds to the conserved "body" momentum density (that is, left translation of $\mathbf{P}$ to the identity).

Dual Pairs. For reasons that are similar to those for incompressible fluids presented in Marsden and Weinstein [1983], the singular solution momentum map $\mathbf{J}_{\text {Sing }}: T^{*} \operatorname{Emb}\left(S, \mathbb{R}^{n}\right) \rightarrow \mathfrak{X}\left(\mathbb{R}^{n}\right)^{*}$ forms one leg of a formal dual pair. We use the words formal dual pair since, in the infinite dimensional case, the proper mathematical underpinnings for the theory of dual pairs has not yet been developed.

Even in the finite dimensional case, there are nontrivial issues to be aware of since dual pairs were studied in the basic paper of Weinstein [1983b]; we refer to Ortega and Ratiu [2004], Chapter 11 as well as Blaom [2001] and Ortega [2003] for background, references and a summary of the current state of the art in this topic. These works show the subtelty of the dual pairs notion, even in finite dimensions, let alone for the infinite dimensional problem we are dealing with here.

The point is that, as we have seen, there is another group that acts on $\operatorname{Emb}\left(S, \mathbb{R}^{n}\right)$, namely the group $\operatorname{Diff}(S)$ of diffeomorphisms of $S$, which acts on the right, while $\operatorname{Diff}\left(\mathbb{R}^{n}\right)$ acted by composition on the left (and this gave rise to our singular solution momentum map, $\left.\mathbf{J}_{\text {Sing }}\right)$. As explained above, the action of $\operatorname{Diff}(S)$ from the right gives us the momentum map $\mathbf{J}_{S}: T^{*} \operatorname{Emb}\left(S, \mathbb{R}^{n}\right) \rightarrow \mathfrak{X}(S)^{*}$. We now assemble both momentum maps into one figure as follows:

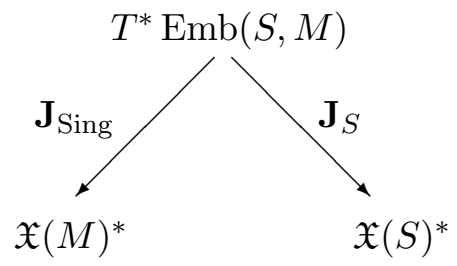

These maps have the formal dual pair property, namely that the kernel of the derivatives of each map at a given point are symplectic orthogonals of one another (see Weinstein [1983a]). Sometimes, as in Ortega and Ratiu [2004], this is called the Lie-Weinstein property .

Formal Proof of the Dual Pair Property. The reduction lemma of Marsden and Weinstein [1974] states that the kernel of the derivative of a momentum map is the symplectic orthogonal of the group orbit. As a consequence, if the group associated to each leg of a potential dual pair of momentum maps acts transitively on the level set of its partner momentum map, then one has a dual pair. In our case, fixing $\mathbf{J}_{S}$ at a value, say $\mathbf{M}$, means, according to equation (5.5), that we fix 
the value of $\mathbf{P} \cdot d \mathbf{Q}$. However, as we already noted above, the group $\operatorname{Diff}(M)$ acts transitively on the space of $\mathbf{Q}$ 's because one can "move the singular surfaces around at will" by means of diffeomorphisms of $M$. The constraint of fixing $\mathbf{P} \cdot d \mathbf{Q}$ is exactly what one needs to transform the $\mathbf{P}$ 's properly by means of the cotangent lift (recall that cotangent lift actions are characterized by preserving the canonical one form). Thus, at least formally, $\operatorname{Diff}(M)$ acts transitively on level sets of $\mathbf{J}_{S}$. Similarly, one sees that $\operatorname{Diff}(S)$ acts transitively on level sets of $\mathbf{J}_{\text {Sing }}$ since fixing $\mathbf{J}_{\text {Sing }}$ corresponds to fixing the image surface, leaving one only with the parametrization freedom, so that $\operatorname{Diff}(S)$ acts transitively on that set.

This is a marvelous framework; it clarifies, amongst other things, the fact that the parameterization of the singular solutions $\mathbf{m}$ in terms of $\mathbf{Q}$ and $\mathbf{P}$ are Clebsch variables in the sense given in Marsden and Weinstein [1983] and that the diffeomorphism group of $S$ corresponds to the gauge group of that Clebsch representation. Also notice that when we write the singular solutions in $\mathbf{Q - P}$ space, we are finding solutions that are collective and so all the properties of collectivization are valid. See Marsden and Ratiu [1999] for a general discussion and references to the original work of Guillemin and Sternberg on this topic.

As explained in Marsden and Weinstein [1983], reduction by the group associated with one leg in a dual pair corresponds to coadjoint orbits on the other leg. Thus, the momentum map $\mathbf{J}_{S}$ captures the analog of the Kelvin circulation theorem of fluid mechanics, as well as its singular version. It would be interesting to explore in more detail this singular analog of the Kelvin circulation theorem for fluids.

The Ideal Fluid Dual Pair. In Marsden and Weinstein [1983] a dual pair was investigated that captures certain singular situations in fluid mechanics, such as the motion of point vortices in the plane. The general dual pair that generalizes that planar case the following:

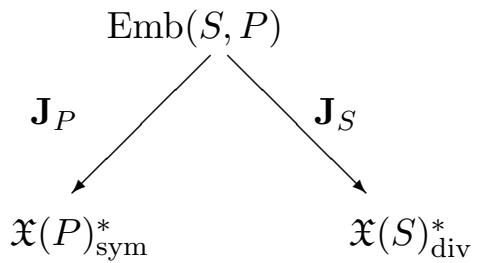

Here, $S$ is a volume manifold, that is a manifold with a volume element, while $P$ is a symplectic manifold. The map $\mathbf{J}_{P}$ is the momentum map for the left action of the group of symplectic diffeomorphisms on $P$, while $\mathbf{J}_{S}$ is the momentum map for the right action of the group of volume preserving diffeomorphisms. This is a very beautiful dual pair, but is not the same as the dual pair we found above of course. In our case, $P=T^{*} M$ was a cotangent bundle and we identified $\operatorname{Emb}(S, P)$ with $T^{*} \operatorname{Emb}(S, M)$. Also, we always dealt with symplectic diffeomorphisms that were cotangent lifts, while in the above dual pair of Marsden and Weinstein [1983], they are general symplectic diffeomorphisms. 
Relation with ideas of Donaldson. Whereas Marsden and Weinstein [1983] focused on ideas related to the dual pair picture, the leg in the above diagram given by the map $\mathbf{J}_{S}$ is discussed from various interesting viewpoints in Donaldson [1999]. In particular, that work (apparently being unaware of the above dual pair of Marsden and Weinstein [1983]) makes a number of intriguing comments, including noting that nontrivial topology can cause the momentum map $\mathbf{J}_{S}$ to be nonequivariant (so there are links with the Calabi theory). Donaldson also studies reduction and makes links with Kähler geometry, hyperKähler geometry, double bracket gradient flows, mean curvature flows, and other intriguing topics. As pointed out by Tony Bloch, there are also interesting links of Donaldson's paper with the Toda flow and factorization problems. We believe that the pursuit of further connections within the circle of ideas for momentum maps should be a source of inspiration for new research in the context of the present paper. For example, this pursuit may lead to new insight into the integrability of the dynamical systems governing these singular solutions.

It seems that we are looking at the tip of a rather large and beautiful iceberg.

\section{Challenges, Future Directions and Speculations}

Numerical Issues: Geometric Integrators. The computations of Martin Staley that illustrated several points in this paper are discussed in detail by Holm and Staley [2003, 2004]. These computations make use of both mimetic differencing and reversibility in a critical way; and this is important for accurate numerical simulations. In other words, integrators that respect the basic geometry underlying the problem obtained accurate singular solutions in numerical simulations. It would be interesting to pursue this aspect further and also incorporate discrete exterior calculus and variational multisymplectic integration methods (see Desbrun, Hirani, Leok and Marsden [2003] as well as Marsden, Patrick and Shkoller [1998] and Lew, Marsden, Ortiz and West [2003]).

Analytical Issues: Geodesic Incompleteness of $H^{1}$ EPDiff. The emergence in finite time of singular solutions from smooth initial data observed numerically in Holm and Staley [2003, 2004] indicates that the diffeomorphism group with respect to the right invariant $H^{1}$ metric is geodesically incomplete when the diffeomorphism group has the $H^{s}$ topology, $s>(n / 2)+1$. The degree of its geodesic incompleteness is not known, but we suspect that almost all EPDiff geodesics in $H^{1}$ cannot be extended indefinitely. This certainly holds in one spatial dimension, where the discreteness of the $\mathrm{CH}$ isospectrum implies that asymptotically in time the $\mathrm{CH}$ solution arising from any confined smooth initial velocity data consists only of peakons. It is an important challenge to find a context in which one can put the $H^{1}$ topology on the diffeomorphism group and reestablish geodesic completeness. The numerics suggests that this might be possible, while known existence theorems, even for the $\mathrm{CH}$ equation are not yet capable of showing this - to the best of our knowledge. 
Reversible Reconnections of the Singular EPDiff Solutions. EPDiff is a reversible equation, and the collisions of its peakon solutions on the line $\mathbb{R}^{1}$ (or the circle $S^{1}$ ) are known to be reversible. The reconnections of the singular EPDiff solutions observed numerically in Holm and Staley [2003, 2004] in periodic domains $\mathbb{T}^{2}$ and $\mathbb{T}^{3}$ are also reversible, and this was used as a test of the numerical method. Reversibility of its reconnections distinguishes the singular solutions of EPDiff from vortex fluid solutions and shocks in fluids, whose reconnections apparently require dissipation and so, are not reversible. The mimetic finite differencing scheme used for the numerical computation of EPDiff solutions in Holm and Staley [2003, 2004] was indeed found to be reversible for overtaking collisions, but it was found to be only approximately reversible for head-on collisions, which are much more challenging for numerical integration schemes.

Applications of EPDiff Singular Solutions in Image Processing. The singular EPDiff solutions correspond to outlines (or cartoons) of images in applications of geodesic flow for the template, or pattern matching approach. The dynamics of the singular EPDiff solutions described by the momentum map (2.8) introduces the paradigm of momentum exchange in soliton collisions into the mechanics and analysis of image processing by template matching. (See Holm, Trouvé, Ratnanather and Younes [2004] for more discussions of this new paradigm for image processing.) First, the momentum representation of the image outlines is non-redundant. That is, the momentum has exactly the same dimension as the matched structures; so there is no redundancy of the representation. Second, the reversibility of the collisions among singular solutions and their reconnections under EPDiff flow assures the preservation of the information contained in the image outlines. In addition, the invariance of the manifold of $N$ singular solutions under EPDiff assures that the fidelity of the image is preserved in the sense of approximation theory. That is, an $N$ soliton approximation of the image outlines remains so, throughout the EPDiff flow. A natural approach for numerically simulating EPDiff flows in image processing is to use multisymplectic algorithms. The preservation of the space-time multisymplectic form by these algorithms introduces an initial-value, final-value formulation of the numerical solution procedure that is natural for template matching.

Rigorous Poisson Structures. In Vasylkevych and Marsden [2003], the question of the (rigorous) Poisson nature of the time $t$ map of the flow of the Euler equations for an ideal fluid in appropriate Sobolev spaces is explored. Given the smoothness properties in $\S 3$, it seems reasonable that similar properties should also hold for the EPDiff equations. However, as mentioned earlier, these smoothness properties do not preclude the emergence of singular solutions from smooth initial data in finite time, because of the possibility for geodesic incompleteness.

Other Groups. The general setting of this paper suggests that perhaps one should look for similar measure valued or singular solutions associated with other problems, including geodesic flows on the group of symplectic diffeomorphisms (relevant for 
plasma physics, as in Marsden and Weinstein [1982]), Bott-Virasoro central extensions and super-symmetry groups.

Scattering. It might be interesting to explore the relation of the singular solution momentum map (2.8) to integrability and scattering data. For example, see Vaninsky [2003] for an interesting discussion of the Poisson bracket for the scattering data of $\mathrm{CH}$ in 1D. This turns out to be the Atiyah-Hitchin bracket, which is also related to the Toda lattice, and this fascinating observation leads to an infinite-dimensional version of Jacobi elliptic coordinates.

Other Issues. Of course there are many other issues remaining to explore that are suggested by the above setting, such as convexity of the momentum map, its extension to Riemannian manifolds, etc. We shall, however, leave these issues for other publications and other researchers.

\section{Acknowledgements}

We are very grateful to Alan Weinstein for his collaboration, help and inspiring discussions over the years. We thank Martin Staley for letting us illustrate some important points using his computations. We also thank Anthony Bloch, Simon Donaldson, Jonathan Munn, Tudor Ratiu, and Richard Thomas for valuable advice and comments.

DDH is grateful for support by US DOE, under contract W-7405-ENG-36 for Los Alamos National Laboratory, and Office of Science ASCAR/AMS/MICS. The research of JEM was partially supported by the California Institute of Technology, the National Science Foundation through the NSF Grant DMS-0204474 and by the Air Force contract F49620-02-1-0176.

\section{References}

Alber, M., R. Camassa, Y. Fedorov, D. Holm and J. E. Marsden [2001], The complex geometry of weak piecewise smooth solutions of integrable nonlinear PDE's of shallow water and Dym type, Comm. Math. Phys. 221, 197-227.

Alber, M. S. and J. E. Marsden [1992], On geometric phases for soliton equations, Commun. Math. Phys. 149, 217-240.

Arnold, V. I. [1966], Sur la géométrie differentielle des groupes de Lie de dimenson infinie et ses applications à l'hydrodynamique des fluids parfaits, Ann. Inst. Fourier, Grenoble 16, 319-361.

Blaom, A. D. [2001], A geometric setting for Hamiltonian perturbation theory, Mem. Amer. Math. Soc. 153, xviii+112.

Camassa, R. and D. D. Holm [1993], An integrable shallow water equation with peaked solitons, Phys. Rev. Lett. 71, 1661-1664, http://xxx.lanl.gov/abs/patt-sol/9305002. 
Camassa, R., D. D. Holm and J. M. Hyman [1994], A new integrable shallow water equation, Adv. Appl. Mech. 31, 1-33.

Camassa, R., D. D. Holm and C. D. Levermore [1996], Long-time effects of bottom topography in shallow water, Physica D, 98 258-286.

Camassa, R., D. D. Holm and C. D. Levermore [1997], Long-time shallow-water equations with a varying bottom, J. Fluid Mech., 349 173-189.

Cantor, M. [1975], Perfect fluid flows over $R^{n}$ with asymptotic conditions, J. Func. Anal. 18, $73-84$.

Courant, T. and A. Weinstein [1988], Beyond Poisson structures. In Action hamiltoniennes de groupes. Troisième théorème de Lie (Lyon, 1986), volume 27 of Travaux en Cours, pages 39-49. Hermann, Paris.

Desbrun, M., A. N. Hirani, M. Leok and J. E. Marsden [2003], Discrete exterior calculus, (preprint).

Donaldson, S. K. [1999], Moment maps and diffeomorphisms, Asian J. Math. 3, 1-15.

Dullin, H. R., G. A. Gottwald and D. D. Holm [2001], An integrable shallow water equation with linear and nonlinear dispersion. Phys. Rev. Lett., 87, 194501-04, http://xxx.lanl. gov/abs/nlin.CD/0104004.

Dullin, H. R., G. A. Gottwald and D. D. Holm [2003] Camassa-Holm, Korteweg-de Vries-5 and other asymptotically equivalent equations for shallow water waves. Fluid Dyn. Res., 33, 73-95.

Dullin, H. R., G. A. Gottwald and D. D. Holm [2004] On asymptotically equivalent shallow water wave equations. Physica D, 190, 1-14.

Ebin, D. G. and J. E. Marsden [1970], Groups of diffeomorphisms and the motion of an incompressible fluid, Ann. of Math. 92, 102-163.

Fringer, O. and D.D. Holm [2001] Integrable vs nonintegrable geodesic soliton behavior. Physica D 150, 237-263, http://xxx.lanl.gov/abs/solv-int/9903007.

Hirani, A., J. E. Marsden and J. Arvo [2001], Averaged template matching equations, Springer Lecture Notes in Computer Science 2134, 528-543.

Holm, D. D. and B. A. Kupershmidt [1983], Poisson brackets and Clebsch representations for magnetohydrodynamics, multifluid plasmas, and elasticity, Physica D 6, 347-363.

Holm, D. D., J. E. Marsden and T. S. Ratiu [1998a], The Euler-Poincaré equations and semidirect products with applications to continuum theories, Adv. in Math. 137, 1-81, http://xxx.lanl.gov/abs/chao-dyn/9801015.

Holm, D. D., J. E. Marsden and T. S. Ratiu [1998b], Euler-Poincaré models of ideal fluids with nonlinear dispersion, Phys. Rev. Lett. 349, 4173-4177.

Holm, D. D., J. E. Marsden and T. S. Ratiu [2002], The Euler-Poincaré equations in geophysical fluid dynamics. In Norbury, J. and I. Roulstone, editors, Large-Scale AtmosphereOcean Dynamics II: Geometric Methods and Models, pages 251-300. Cambridge Univ. Press. 
Holm, D. D., J. E. Marsden, T. S. Ratiu and A. Weinstein [1985], Nonlinear stability of fluid and plasma equilibria, Phys. Rep. 123, 1-196.

Holm, D. D., V. Putkaradze and S. N. Stechmann [2003], Rotating concentric circular peakons. Submitted to Nonlinearity, http://arxiv.org/abs/nlin.SI/0312012/ 9801015.

Holm, D. D., J. T. Ratnanather, A. Trouvé and L. Younes [2004], Solitons in computational anatomy. To appear in NeuroImage.

Holm, D. D. and M. F. Staley [2003], Wave structures and nonlinear balances in a family of evolutionary PDEs. SIAM J. Appl. Dyn. Syst. 2, 323-380.

Holm, D. D. and M. F. Staley [2004], Momentum dynamics of filaments and surfaces in a set of $2+1$ and $3+1$ evolutionary PDEs. In preparation.

Kodama, Y. [1985], On integrable systems with higher order corrections Phys. Lett. A 107, $245-249$.

Kodama, Y. [1985a], Normal forms for weakly dispersive wave equations Phys. Lett. A 112, 193-196.

Kodama, Y. [1987], On solitary-wave interaction Phys. Lett. A 123, 276-282.

Kruse, H.P., J. Scheurle and W. Du [2001], A two-dimensional version of the CH equation. In Symmetry and Perturbation Theory: SPT 2001 Edited by D. Bambusi, G. Gaeta and M. Cadoni. World Scientific: New York, 120-127.

Lew, A., J. E. Marsden, M. Ortiz and M. West [2003], Asynchronous variational integrators, Archive for Rat. Mech. An. 167, 85-146.

Liu, A. K., Y.S. Chang, M. K. Hsu and Liang, N. K., [1998], Evolution of nonlinear internal waves in East and South China Seas, J. Geophys. Res., 103, 7995-8008.

Marsden, J. E. and T. J. R. Hughes [1983], Mathematical Foundations of Elasticity. Prentice Hall. Reprinted by Dover Publications, NY, 1994.

Marsden, J. E., G. W. Patrick and S. Shkoller [1998], Multisymplectic geometry, variational integrators and nonlinear PDEs, Comm. Math. Phys. 199, 351-395.

Marsden, J. E. and T. S. Ratiu [1999], Introduction to Mechanics and Symmetry, volume 17 of Texts in Applied Mathematics, vol. 17; 1994, Second Edition, 1999. Springer-Verlag.

Marsden, J. E., T. Ratiu and S. Shkoller [2000], The geometry and analysis of the averaged Euler equations and a new diffeomorphism group, Geom. Funct. Anal. 10, 582-599.

Marsden, J. E., T. S. Ratiu and A. Weinstein [1984], Semi-direct products and reduction in mechanics, Trans. Amer. Math. Soc. 281, 147-177.

Marsden, J. E. and A. Weinstein [1974], Reduction of symplectic manifolds with symmetry, Rep. Math. Phys. 5, 121-130.

Marsden, J. E. and A. Weinstein [1982], The Hamiltonian structure of the Maxwell-Vlasov equations, Physica D 4, 394-406. 
Marsden, J. E. and A. Weinstein [1983], Coadjoint orbits, vortices and Clebsch variables for incompressible fluids, Physica D 7, 305-323.

Miller, M.I., A. Trouvé and L. Younes [2002], On the metrics and Euler-Lagrange equations of computational anatomy. Annu. Rev. Biomed. Eng. 4, 375-405.

Mumford, D. [1998], Pattern theory and vision. In Questions Matheématiques En Traitement Du Signal et de L'Image, Chapter 3, pp. 7-13. Paris: Institut Henri Poincaré (1998).

Ortega, J.-P. [2003], Singular dual pairs, Differential Geom. Appl. 19, 61-95.

Ortega, J.-P. and T. S. Ratiu [2004], Momentum maps and Hamiltonian reduction, volume 222 of Progress in Mathematics. Birkhäuser Boston Inc., Boston, MA.

Shkoller, S. [1998], Geometry and curvature of diffeomorphism groups with $H^{1}$ metric and mean hydrodynamics, J. Funct. An. 160, 337-365.

Vaninsky, K. L. [2002], The Atiyah-Hitchin bracket and the open Toda lattice, ArXiv:mathph/0202047 v1. To appear in J. Geom. and Phys.

Vaninsky, K. L. [2003], The Camassa-Holm equation on the line and Jacobi elliptic coordinates, ArXiv:math-ph/0303063.

Vasylkevych, S. and J.E. Marsden [2003], The Lie-Poisson structure of the Euler equations of an ideal fluid. (preprint).

Weinstein, A. [1971], Symplectic manifolds and their Lagrangian submanifolds, Adv. Math. 6, 329-346. see also Bull. Am. Math. Soc. 75 (1969), pp. 1040-1041.

Weinstein, A. [1973], Normal modes for nonlinear Hamiltonian systems, Inv. Math. 20, $47-57$.

Weinstein, A. [1977], Lectures on symplectic manifolds, volume 29 of CBMS Regional Conf. Ser. in Math. Conf. Board Math. Sci., Washington, DC.

Weinstein, A. [1978], Bifurcations and Hamilton's principle, Math. Zeit. 159, 235-248.

Weinstein, A. [1983a], Sophus Lie and symplectic geometry, Expo. Math. 1, 95-96.

Weinstein, A. [1983b], The local structure of Poisson manifolds, J. of Diff. Geom. 18, 523557 .

Weinstein, A. [1984], Stability of Poisson-Hamilton equilibria, Contemp. Math. 28, 3-14.

Weinstein, A. [1990], Connections of Berry and Hannay type for moving Lagrangian submanifolds, Adv. in Math. 82, 133-159.

Weinstein, A. [1996], Lagrangian Mechanics and Groupoids, Fields Inst. Commun. 7, 207231.

Weinstein, A. [2002], Geometry of momentum (preprint); ArXiv:math/SG0208108 v1.

Xin, Z. and P. Zhang [2000], On the weak solutions to a shallow water equation, Comm. Pure Appl. Math. 53, 1411-1433. 